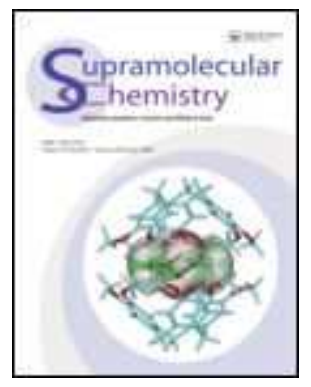

\title{
Influence of laterally attached alkyl groups on the conformational behaviour of a basic calix [4]arene: Combined NMR, Molecular Mechanics and X-ray study
}

\begin{tabular}{|r|l|}
\hline Journal: & Supramolecular Chemistry \\
\hline Manuscript ID: & GSCH-2009-0016.R2 \\
\hline Manuscript Type: & Full Paper \\
\hline Date Submitted by the & $20-$ Oct-2009 \\
\hline Complete List of Authors: & $\begin{array}{l}\text { Weber, Edwin; TU Bergakademie Freiberg, Organische Chemie } \\
\text { Gruner, Margit; TU Dresden, Chemistry } \\
\text { Gruber, Tobias; University of Freiburg, Pharmaceutical Sciences } \\
\text { Fischer, Conrad; TU Bergakademie Freiberg, Organic Chemistry }\end{array}$ \\
\hline Keywords: & $\begin{array}{l}\text { calixarenes, lateral substitution, synthesis, conformational study, X- } \\
\text { ray crystal structure }\end{array}$ \\
\hline $\begin{array}{l}\text { Note: The following files were submitted by the author for peer review, but cannot be converted } \\
\text { to PDF. You must view these files (e.g. movies) online. }\end{array}$ \\
\hline $\begin{array}{l}\text { guish3.cif } \\
\text { checkCIF_PLATON report.eml }\end{array}$ \\
\hline \hline
\end{tabular}

\section{S ScholaroNE \\ Manuscript Central}


Horizontal broadening: Calix[4]arenes featuring the structural mode of lateral alkyl substituents were prepared. As proven by special ROESY- and EXSY-2D-NMR experiments, the lateral alkyl group substituents are not exercising a decisive influence on the conformational behaviour of the parent calixarene, suggesting a promising approach for horizontal expansion of the calixarene platform.

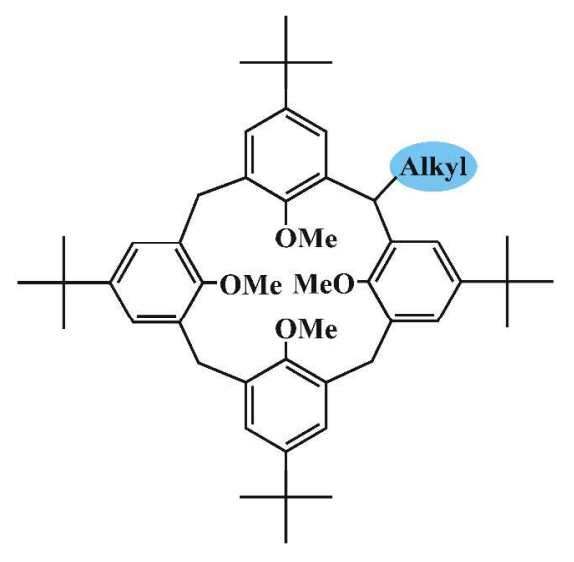

M. Gruner, C. Fischer, T. Gruber,

E. Weber*.

Influence of laterally attached alkyl groups on the conformational behaviour of a basic calix[4]arene: Combined NMR, Molecular Mechanics and X-ray study 


\title{
Influence of laterally attached alkyl groups on the conformational behaviour of a basic calix[4]arene: Combined NMR, Molecular Mechanics and X-ray study
}

\author{
Margit Gruner $^{\mathrm{a}}$, Conrad Fischer ${ }^{\mathrm{b}}$, Tobias Gruber $^{\mathrm{b}}$ and Edwin Weber ${ }^{\mathrm{b} *}$ \\ a Institut für Organische Chemie, Technische Universität Dresden, Mommsenstr. 13 \\ D-01062 Dresden, Germany; ${ }^{\mathrm{b}}$ Institut für Organische Chemie, Technische Universität \\ Bergakademie Freiberg, Leipziger Str. 29, D-09596 Freiberg/Sachs., Germany
}

* Corresponding author. Email: edwin.weber@chemie.tu-freiberg.de 
Three new calixarenes 3-5 featuring an alkyl residue of different chain length attached to one of the central ring methylene groups of the basic calix[4]arene $\mathbf{1}$ have been prepared. A systematic study that includes also the lower homologous compound 2 , showing the effect of the alkyl substitution on the conformational behaviour of the calixarene framework in comparision with the unsubstituted parent compound $\mathbf{1}$ is reported. The application of special 2D NMR techniques, 2D-EXSY and ROESY method, at various temperatures establishes that the calixarenes $\mathbf{2 - 5}$ adopt the partial cone conformation of lower symmetry and far less the symmetric cone and 1,2-alternate conformations. In solution, they undergo a fast interconversion with relatively low activation energies of about $15 \mathrm{kcal} / \mathrm{mol}$ at room temperature. The conformer distribution is reproduced well by molecular mechanistic calculations (MMFF94) indicating the present conformers to assume the lowest steric energies. A single-crystal X-ray structure of the lateral ethyl derivative $\mathbf{2}$ corroborates these results, showing the molecule in a sterically favourable partial cone conformation.

Keywords: calixarenes; lateral substitution; synthesis; conformational study; solution 2D NMR measurements; X-ray crystal structure

\section{Introduction}

Calixarenes and their derivatives are an important class of compounds that have been extensively investigated regarding their supramolecular recognition properties during the past two decades (1). Calixarenes have also emerged as very attractive building blocks for the development of highly ingenious functional systems and devices (2). In both of these respects, much work has been dedicated to the synthesis of calixarenes modified on the upper and lower rims, giving rise to an enormous variety of compound 
structures (3). Homocalixarenes (4), thiacalixarenes (5) and others (1) where the bridging methylene units of the conventional calixarene framework are completely replaced by modifying organic groups or hetero atoms, represent further currently studied yet rather well known types of structural modifications of the calixarene family (6). By way of contrast, calixarenes featuring a substitution of only one of the methylene bridges are presently rare (7), although these laterally substituted calixarenes may open interesting aspects of calixarene chemistry including linked constructions of oligotopic receptors (8) as well as systems distinguished by particular anchoring of the calixarene to a specific target or support $(2,9)$. As a result, the conformational behaviour of this particular compound type is also studied only fragmentary in a few cases of modified tetrahydroxycalix[4]- and -calix[5]arenes $(7 c-d)$. This warrants to deal with the conformational property of laterally monosubstituted calixarenes more broadly. Hence, a series of calixarenes that feature a lateral substitution of alkyl groups of different chain length and includes a well-known parent compound was considered for this purpose. The concrete system of compounds which was chosen for this study, as specified in Fig. 1, covers the tetra-tert-butyltetramethoxycalix[4]arene (1) as the reference and $\mathbf{2 - 5}$ as the test compounds. This shows an advantage since the conformational behaviour of $\mathbf{1}$ is known rather detailed from previous work (10). Moreover, for the synthesis of 2-5 one can follow a literature method elaborated for the calixarene 2 by Scully (7a). The conformational properties of 2-5, which are described here, were determined from a detailed solution NMR study. This involves measurements at various temperatures and uses special 2D NMR techniques to yield a full description of the conformer distribution and the free activation energies of conformational interconversion. We also report on the crystal structure of the corresponding laterally ethyl monosubstituted calixarene $\mathbf{2}$, being the first crystal structure of a laterally monosubstituted tetramethoxycalix[4]arene. 


\section{Results and discussion}

\section{Synthesis}

The calixarene $\mathbf{1}$, which is the basic compound for the synthesis of $\mathbf{2 - 5}$, was obtained from two-stage methylation reaction of 5,11,17,23-tetra-tert-butyl-25,26,27,28tetrahydroxycalix[4]arene (11) using in a starting phase-transfer process sodium hydroxide, dimethyl sulphate and tetrabutylammonium bromide to yield the intermediate compound with two distal methyl groups, followed by treatment with sodium hydride and iodomethane in a final methylation step (12). A corresponding onestep methylation method of the parent tetrahydroxycalixarene with sodium hydride and iodomethane, described in the literature $(10 a, 13)$, did not prove competitive in its result. The target calixarenes $\mathbf{2 - 5}$ featuring different laterally substituted alkyl groups were prepared by deprotonation of $\mathbf{1}$ with $n$-butyllithium and reaction with respective alkyl halides following the procedure described by Scully $(7 a)$.

\section{Conformational study}

Conformational predominance and conformational flexibility are important structural parameters of a calixarene molecule, being decisive of its supramolecular behaviour both in solution and solid state (1-3). While the so-called cone conformation, providing a spaceous cavity structure, is perhaps the most frequent conformation of the existing calixarenes $(1,14)$, the other types of molecular conformations, including partial cone, 1,2- and 1,3-alternate conformation, are somewhat more rarely found though not less important for a designed purpose (15). As a rule, these conformations are mainly determined by the ring size or the nature and the space required of the substituents being present at the upper and lower rims of the calixarene molecule. Conformational property 
will also depend on the physical condition, including type of solvent as well as the presence of a proper guest molecule. However, in no manner, $O$-alkylated calixarenes that feature an alkyl residue laterally attached to one of the methylene groups of the central ring have been studied conformationally. Only analogous tetrahydroxycalixarenes were taken for a corresponding study $(7 c-d)$.

A well-known molecular structure is that of the tetra-tertbutyltetramethoxycalix[4]arene (1), which was chosen as the parent compound for the test. According to the NMR data (10), 1 in a solution of $\mathrm{CDCl}_{3}$ exists in an equilibrium of all four conventional conformers of which the partial cone conformation is the preferred one. A partial cone conformer is also reported for the solvent free calixarene $\mathbf{1}$ (16) as well as for the furan (17) and dichloromethane (18) inclusion compounds in the solid state, while a corresponding sodium complex shows a cone conformation both in the crystal structure and in $\mathrm{CDCl}_{3} / \mathrm{CD}_{3} \mathrm{CN}$ solution due to coordination of the sodium ion $(10 b)$. Thus, it is clear that a potential change of the conformational behaviour in the present cases of calixarenes, in comparison with $\mathbf{1}$, is attributed to the laterally substituted alkyl groups.

A complex pattern of signals in the NMR spectra of the alkyl substituted calixarenes 2-5 in both $\mathrm{CDCl}_{3}$ and tetrachloroethane- $\mathrm{d}_{2}\left(\mathrm{TCl}-\mathrm{d}_{2}\right)$ at various temperatures indicates that the compounds exist in different conformers, as demonstrated exemplary for the lateral propyl derivative $\mathbf{3}$ in Fig. 2. To minimize the signal broadening and separate the overlapping region of methine and methylene signals, we carried out the NMR experiments at an optimized temperature of $269 \mathrm{~K}$, yielding three different signals for the methine protons between 4.47 and $4.79 \mathrm{ppm}$ in $\mathrm{CDCl}_{3}$ for compounds 2-5. Detailed 2D NMR measurements using COSY-, ${ }^{1} \mathrm{H} /{ }^{13} \mathrm{C}$-correlated HSQC (19) and HMBC (20) as well as NOESY and ROESY experiments gave a full determination of conformers (specified in Fig. $3 ;{ }^{1} \mathrm{H}$ and ${ }^{13} \mathrm{C}$ chemical shifts are summarized in Tables 
$1 \mathrm{~S}-4 \mathrm{~S}$ of the Supplementary Information). A special denotation of the calixarene scaffold in the three observed conformations is illustrated in Fig. 3, indicating similarities in the chemical shift for different pairs of atoms.

Depending on the alignment of the four arene units with reference to the lateral substituent, there are 16 different conformational isomers possible (Fig. 4). Six of them are mirror images of each other resulting in identical NMR spectra. The 2D experiments show that the laterally substituted $n$-alkyl calixarenes, studied here, exist mainly in one partial cone conformation (pacoD in Fig.4), with an equatorial substituent between two syn orientated arene units, and also as 1,2-alternate (alt $2 \mathrm{C}$ in Fig. 4) and cone (coneB in Fig. 4) conformers (Scheme 1), revealed in the region of the $\mathrm{OCH}_{3}$ groups of the HSQC spectrum (Fig. 5). In the ROESY spectrum (Fig. 6), all conformers possess stronger positive cross peaks between the axial methine $\mathrm{H}-1$ protons in the bridge and the near H-8 protons of the methoxy groups than between these protons and the $\mathrm{H}-3$ protons of the same aromatic rings, indicating the vicinity of one of the methoxy groups on the lower rim to $\mathrm{H}-1$. Further characteristic specific interactions between aromatic protons of the calixarene and protons of $\mathrm{OCH}_{3}$ or tert-butyl groups neighbouring the methylene bridge are marked in Fig. 6. These correlations suggest the orientation of arene units of the calixarene ring for each individual isomer. For example, the paco isomer is characterized by the cross peaks $8 \mathbf{\Delta}^{\prime} / 11 \mathbf{\Delta}^{\prime}, 16 \mathbf{\Delta} / 13 \mathbf{\Delta}, 16 \mathbf{\Delta}^{\prime} / 5 \mathbf{\Delta}^{\prime}$ and $16 \mathbf{\Delta}^{\prime} / 13 \mathbf{\Delta}$, and the 1,2-alternate species by the $8 \boldsymbol{\square} / 11 \boldsymbol{\square}$ and $8 \boldsymbol{\square} / 13 \boldsymbol{\square}$ relation.

Furthermore, in Fig. 6, negative cross-peaks between different H-1 proton signals can be observed due to chemical exchange processes in the form of a dynamic interconversion of conformers. To extract the rate constants for the confomational chemical exchange $\left(k_{\text {chem }}\right)$, the 2D-EXSY method (21) was used, and thus the free activation energies $\Delta G^{\#}$ of the interconversion has been calculated (Table 1). Interestingly, compounds 2-5 display a fast partial cone/cone interconversion involving 
values of free activation energy rather comparable to the reported $\sim 13 \mathrm{kcal} / \mathrm{mol}$ for the laterally unsubstituted prototype calixarene $\mathbf{1}$ in the same solvent $(10 a)$. The observed influence of solvent and temperature on the conformational behaviour is in agreement with the common expectation. In this connection, the conformational reorientation was found to be faster at higher temperature and retarded in solution of the larger and less polar solvent molecules of tetrachloroethane- $\mathrm{d}_{2}\left(\mathrm{TCl}-d_{2}\right)$ compared to $\mathrm{CDCl}_{3}$.

The conformer distribution of $\mathbf{1}$ and $\mathbf{2 - 5}$, summarized in Table 2, shows that the partial cone conformation predominates the present series of tetra-tertbutyltetramethoxycalix[4]arenes in $\mathrm{CDCl}_{3}$, varying only little by introduction of the lateral alkyl substituents, i.e. the predominance of the partial cone conformation is only slightly affected. Nevertheless, its frequency decreases to some extent for the benefit of the rarely occurring cone- and 1,2-alternate structures in the following manner. With increasing chain length, the ratio of partial cone conformation remains nearly unchanged, while the cone conformation decreases and the 1,2-alternate conformation increases. However, as contrasted with the prototype calixarene $\mathbf{1}$, for none of the studied compounds a 1,3-alternate conformation was detected. This points to a predominance of thermodynamically stable conformers and suggests the avoidance of an isoclinal arrangement of the lateral substituent, presumably for energetic reasons.

\section{Calculation}

To evaluate the steric influence of the lateral alkyl substituent, relative energies of the different conformers of $\mathbf{2}$ as a model compound, summarized in Fig. 4, were determined using the MMFF94 force field (22) as implemented in the SYBYL software. As proven by previous mechanistic studies $(7 d)$, all conformations with an axial arrangement of the lateral substituent are energetically discriminated due to their higher angle bending 
energy. The predominance of the partial cone conformation resulting from the NMR experiments, bearing the lateral substituent in an equatorial position between syn orientated arene units in solution (pacoD) may thus be explained with the avoidance of repulsion between the $\alpha$-C-atom of the lateral alkyl moiety and the neighbouring methoxy groups, indicated by their low sterical energies (Table 3). Analogously, the frequency and stability of the coneB conformer detected by NMR can be assumed due to its similar low ground state energy. It is also shown that the energy gap between the 1,2-alternate (alt2C) and the 1,3-alternate conformer is small, giving evidence of their potential presence in solution. The fact that only the 1,2-alternate conformer is detected in the NMR could result from its higher energy gap to the lowest energy partial cone conformer ( $3.8 \mathrm{kcal} / \mathrm{mol}$ for alt $2 \mathrm{C})$ leading to separate signals, whereas the interconversion to the 1,3-alternate conformer is too fast on the NMR time scale.

\section{Crystal structure of compound $2 \cdot \mathrm{CHCl}_{3}$}

In order to compare the conformational results from the solution NMR study with the conformational behaviour in the solid state, we tried to obtain suitable crystals of the compounds 2-5 for X-ray study. Unfortunately, we were unsuccessful in getting highquality crystals in the case of $\mathbf{3 - 5}$, presumably due to the high flexibility of the rather extended alkyl chains. However, the corresponding laterally ethyl substituted compound 2 yielded colourless crystals (space group $P c a 2_{1}$ ) from a solvent mixture of methanol and chloroform (2:1) suitable for X-determination (Table 4). The crystals were analyzed to be a stoichiometric 1:1 solvate with chloroform. Not surprisingly, also in the solid state, one low energy partial cone conformer bearing the lateral substituent in an equatorial position between syn orientated arene units is found (Fig. 7). This result is in agreement both with the predominance of the partial cone conformation in solution and the lowest energy conformer ( $p a c o D)$ from MM calculation. 
Selected conformational parameters of the calixarene molecule $\mathbf{2}$ are summarized in Tab. S5. The opposite phenyl rings A and C differ only little from coplanarity [8.9 ${ }^{\circ}$ (1)], while the aromatic rings B and D are orientated in an angle of $42.8^{\circ}$ (1). Similar to a comparable 2:1 solvate of the lateral unsubstituted calixarene 1 with dichloromethane (18), the methoxy group of ring D points outwards and maintains no interactions with the other rings. In consequence of the compact structure caused by the partial cone conformer, the calixarene does not allow a cavitate inclusion of solvent molecules, which therefore are located interstitially in the crystal lattice. With reference to the crystal packing, the calixarene molecules are orientated translatory staggered along the crystallographic $b$ axis (Fig. 8). Corresponding to the nonpolar character of the calixarene, the interactions are limited to weak $\mathrm{C}-\mathrm{H}^{\cdots} \pi$-contacts $(23)$ involving guest and calixarene molecules $\left[d\left(\mathrm{C} 1 \mathrm{GA}^{\cdots}\right.\right.$ centroid $\mathrm{C}=3.569$ (1) $\AA$ ) or the calixarene molecules among each other $[d(\mathrm{C} 33 \cdots$ centroid $\mathrm{B}=3.486(1) \AA]$ as specified in Tab. S6.

\section{Conclusions}

According to the literature $(1,7)$, calixarenes showing a substitution of a single methylene bridge, especially in the case of an alkyl substitution, are a rather low developed class of compounds. As derivatives, of the archetype tetramethoxycalix[4]arene $\mathbf{1}$, only the first two members of the potential compound series, i.e. the methyl- and ethyl (2) derivatives have previously been synthesized and preliminarily described regarding their conformational behaviour. With the present calixarenes 3-5 featuring the corresponding mode of lateral substitution but using higher alkyl groups of different chain length, new examples of this particular compound type have now been made available. They enable to perform a first systematic study of the 
influence of the given alkyl groups on the conformational property of the tetramethoxycalix[4]arene system.

The following main conclusions can be obtained from this study: (1) The lateral attachment of an alkyl residue to one of the central ring methylene groups of the basic calixarene $\mathbf{1}$ has no substantial consequence on the conformational behaviour. That is, independent of the chain length, a respective substitution of an alkyl group does not hamper the predominance of the partial cone conformation of $\mathbf{1}$ in chloroform solution, as proven by NMR spectroscopy. This particular conformation was also found to be low energetic in the calculated sterical energies. Moreover, the crystal structure of compound $\mathbf{2}$ in its solvated form with chloroform is in structural agreement, showing the partial cone conformation in the crystalline state. (2) As against the unsubstituted calixarene 1, the free activation energy for the conformational interconversion of $\mathbf{2 - 5}$ in solution is found only moderately higher. Hence, it is shown that (3) an alkyl substitution of one of the hydrogen atoms of a methylene group between the flanking aryl residues of the central ring offers a very promising possibility for coupling of a basic tetramethoxy-calix[4]arene structural element to spacer or linker units in order to realize future developments of new complex framework structures without substantial impairment of the initial calixarene conformation, while corresponding modifications at the upper and lower rim sites normally result in distinct changes of the conformational behaviour.

Future work is required to explore the effect of more than one laterally attached alkyl groups or of a substituent containing polar or functional units in the side chain. Investigations into this direction are aiming work of our group.

\section{Experimental}




\begin{abstract}
Materials
1-Iodopropane (Fluka), 1-iodopentane (Acros) and 1-bromodecane (Fluka) were used as received. The $n$-BuLi (1.6 M in hexane) was purchased from Acros. Analytical TLC was performed on precoated silica gel plates (Merck, $60 \mathrm{~F}_{254}$ ). Organic solvents were purified by standard procedures (24).
\end{abstract}

\title{
Techniques
}

Melting points were determined with a hot-stage microscope (VEB Dresden Analytik) and are uncorrected. ${ }^{1} \mathrm{H}$ and ${ }^{13} \mathrm{C}$ NMR spectra were recorded using a Bruker DRX 500 spectrometer. The chemical shifts $(\delta)$ are reported in ppm relative to TMS $=0 \mathrm{ppm}$. Mass spectra were recorded with an EI-MS (Finnigan MAT 8200). The elemental analyses were performed with a Heraeus $\mathrm{CHN}$ rapid analyzer.

\section{Synthesis of 5,11,17,23-tetra-tert-butyl-25,26,27,28-tetramethoxycalix[4]arene (1)}

A mixture of 5,11,17,23-tetra-tert-butyl-25,26,27,28-tetrahydroxycalix[4]arene (11), $\mathrm{NaOH}$ (aqueous, 50 p.c.), dimethyl sulphate and tetrabutylammonium bromide in toluene was reacted following a known procedure (12). Recrystallization from $\mathrm{MeOH} / \mathrm{CHCl}_{3}$ (1:1) yielded $60 \%$ of intermediate 5,11,17,23-tetra-tert-butyl-26,28dihydroxy-25,27-dimethoxycalix[4]arene as colorless solid, m.p. 235-238 ${ }^{\circ}$ Following the literature protocol (13), this compound was caused to react with $\mathrm{NaH}$ and iodomethane to afford $67 \%$ of calixarene $\mathbf{1}$ as a colorless solid, m.p. 242-244 ${ }^{\circ} \mathrm{C}$ (lit.(12) $242-243{ }^{\circ} \mathrm{C}$ ).

General procedure for the synthesis of the laterally monoalkylated calix[4]arenes 2-5 
The synthesis of the products $\mathbf{2 - 5}$ follows the protocol described by Scully et al. (7a). To a stirred solution of $\mathbf{1}(1.0 \mathrm{mmol})$ in dry THF (30 ml), $n$-BuLi (1.6 $\mathrm{N}$ in $n$-hexane, 2.9 $\mathrm{ml}, 4.6 \mathrm{mmol}$ ) was added at room temperature under argon. After having stirred for 30 min, the respective 1-halogenalkane $(8.0 \mathrm{mmol})$ was added via syringe to the solution, causing a change of its color from red to yellow. Stirring was continued for $1 \mathrm{~h}$. The solvent was removed under reduced pressure and the residue partitioned into water/dichloromethane $(1: 1,60 \mathrm{ml})$. The organic layer was washed with water $(2 \mathrm{x}$ $30 \mathrm{ml})$, dried $\left(\mathrm{MgSO}_{4}\right)$ and evaporated under reduced pressure. Recrystallization from $\mathrm{MeOH}$ yielded the products as colorless solids. Experimental, MS and elemental analysis data of the individual compounds are specified below. For the detailed 2D NMR experiments, all substances were dried for $24 \mathrm{~h}$ under vacuum at $50{ }^{\circ} \mathrm{C}$. The respective NMR data of $\mathbf{2 - 5}$ are given in the Supplementary Materials (Tables S1-S4). The NMR data for 2 correspond to the literature (7a).

\title{
5,11,17,23-Tetra-tert-butyl-25,26,27,28-tetramethoxy-2-ethylcalix[4]arene (2)
}

1-Bromoethane was used. Yield $0.42 \mathrm{~g}(57 \%), \mathrm{mp} .183-185^{\circ} \mathrm{C}$ [lit. (7a) 184-186 $\left.{ }^{\circ} \mathrm{C}\right]$.

\author{
5,11,17,23-Tetra-tert-butyl-25,26,27,28-tetramethoxy-2-n-propylcalix[4]arene (3) \\ 1-Iodopropane was used. Yield $0.34 \mathrm{~g}(46 \%)$, mp. $132-135{ }^{\circ} \mathrm{C}$. Anal. Calcd for \\ $\mathrm{C}_{51} \mathrm{H}_{70} \mathrm{O}_{4} \cdot 0.5 \mathrm{MeOH}: \mathrm{C} 81.06, \mathrm{H} 9.51$. Found: C 80.99, H 9.78 \%. MS (70 eV), m/z \\ (\%): $747\left(\mathrm{M}^{+}, 100\right), 748(52), 358(35), 177(28)$.
}

\section{5,11,17,23-Tetra-tert-butyl-25,26,27,28-tetramethoxy-2-n-pentylcalix[4]arene (4)}

1-Iodopentane was used. Yield $0.35 \mathrm{~g}(47 \%)$, mp. $76-80{ }^{\circ} \mathrm{C}$. Anal. Calcd for $\mathrm{C}_{53} \mathrm{H}_{74} \mathrm{O}_{4}$ :

C 82.12, H 9.62. Found: C 82.15, H 9.80 \%. MS (70 eV), $m / z(\%): 775\left(\mathrm{M}^{+}, 100\right), 776$ (64), 372 (43), 177 (14). 


\author{
5,11,17,23-Tetra-tert-butyl--25,26,27,28-tetramethoxy-2-n-decylcalix[4]arene (5) \\ 1-Bromodecane was used. Yield $0.37 \mathrm{~g}$ (44\%), mp. 139-144 ${ }^{\circ} \mathrm{C}$. Anal. Calcd for \\ $\mathrm{C}_{58} \mathrm{H}_{84} \mathrm{O}_{4} \cdot 1.5 \mathrm{MeOH}$ : C 80.00, H 10.15. Found: C 79.85, H 9.88 \%. MS (70 eV), m/z \\ (\%): $845\left(\mathrm{M}^{+}, 100\right), 846(63), 407$ (42), 177 (32).
}

\title{
X-ray crystallography
}

Crystals of $\mathbf{2}$ suitable for X-ray diffraction analysis were obtained by slow recrystallization from methanol/chloroform $(2: 1)$ solution. The intensity data were collected on a Bruker APEX II diffractometer with $\operatorname{MoK}_{\alpha}$ radiation $(\lambda=0.71073 \AA)$ using $\omega$ - and $\varphi$-scans. Reflections were corrected for background and Lorentz polarization effects. Preliminary structure models were derived by application of direct methods (25) and were refined by full-matrix least squares calculation based on $F^{2}$ for all reflections (26). All hydrogen atoms were included in the models in calculated positions and were refined as constrained to bonding atoms. Relevant crystal data together with the refinement details are listed in Table 4.

Crystallographic data for the structural analysis have been deposited with the Cambridge Crystallographic Data Centre, No. CCDC 748215. Copies of this information may be obtained free of charge from: The Director, CCDC, 12 Union Road, Cambridge, CB2 1EZ, UK. Fax (+44) 1223-336-033; E-mail: deposit@ccdc.cam.ac.uk or www: www.ccdc.cam.ac.uk. 


\section{Supplementary materials}

${ }^{1} \mathrm{H}$ and ${ }^{13} \mathrm{C}$ NMR data of $\mathbf{2 - 5}$ in $\mathrm{CDCl}_{3}$ and tetrachloroethane- $\mathrm{d}_{2}$, selected conformational parameters and geometric parameters of potential hydrogen bond type interactions from the crystal structure of $\mathbf{2} \cdot \mathrm{CHCl}_{3}$ are available as Supplementary Materials, available online.

\section{Acknowledgements}

This work was supported by the German Federal Ministry of Economics and Technology (BMWi) under grant No. 16IN0218 'ChemoChips'. The authors are also grateful to Dr. I. Thondorf for help with the MM calculation. 


\section{Reference}

(1) Gutsche, C. D. Calixarenes - An Introduction, 2nd edn; Royal Society of Chemistry: Cambridge, UK, 2008.

(2) Baklouti, L.; Vicens, J.; Harrowfield, J. In Calixarenes in the Nanoworld; Springer: Dordrecht, 2007.

(3) Asfari, M. Z; Böhmer V.; Harrowfield, J.; Vicens, J. In Calixarenes 2001; Kluwer: Dordrecht, 2001.

(4) Ibach, S.; Prautzsch, V.; Vögtle, F.; Chartroux, C.; Gloe, K. Acc. Chem. Res. 1999, 32, 729-740.

(5) Morohashi, N.; Narumi, F.; Iki, N.; Hattori, T.; Miyano, S. Chem. Rev. 2006, $106,5291-5316$.

(6) For recent studies, see: (a) Columbus, I.; Biali, S. E. J. Org. Chem. 2008, 73, 2598-2606.; (b) Columbus, I.; Biali, S. E. Org. Lett. 2007, 9, 2927-2929.

(7) (a) Scully, P. A.; Hamilton, T. M.; Bennett, J. L. Org. Lett. 2001, 3, 2741-2744;

(b) Middel, O.; Greff, Z.; Taylor, N. J.; Verboom, W.; Reinhoudt, D. N.; Snieckus, V. J. Org. Chem. 2000, 65, 667-675; (c) Bergamaschi, M.; Bigi, F.; Lanfranchi, M.; Maggi, R.; Pastorio, A.; Pellinghelli, M. A.; Peri, F.; Porta, C.; Satori, G. Tetrahedron 1997, 53, 13037-13052; (d) Biali, S. E.; Böhmer, V.; Cohen, S.; Ferguson, G.; Grüttner, C.; Grynspan, F.; Paulus, E. F.; Thondorf, I.; Voigt, W. J. Am. Chem. Soc. 1996, 118, 12938-12949.

(8) (a) Podoprygorina, G.; Janke, M.; Janshoff, A.; Böhmer, V. Supramol. Chem. 2008, 20, 59-69; (b) Breitkreuz, C. J.; Zadmard, R.; Schrader, T. Supramol. Chem. 2008, 20, 109-115; (c) Othman, A. B.; Lee, Y. H.; Ohto, K.; Abidi, R.; Kim, Y.; Vicens, J. J. Incl. Phenom. Macrocycl. Chem. 2008, 62, 187-191; (d) Baklouti, L.; Cheriaa, N.; Mahouachi, M.; Abidi, R.; Kim, J. S.; Kim, Y.; Vicens, J. J. Incl. Phenom. Macrocycl. Chem. 2006, 54, 1-7; (e) Garozzo, D.; Gattuso, G.; Notti, A.; Pappalardo, A.; Pappalardo, S.; Parisi, M. F.; Perez, M.; Pisagatti, I. Angew. Chem. 2005, 117, 4970-4974; (Angew. Chem. Int. Ed. 2005, 44, 4892-4896).

(9) (a) Costa, A. I.; Prata, J. V. Supramol. Chem. 2008, 20, 95-108; (b) Jain, V. K.; Pandya, R. A.; Pillai, S. G.; Shrivastav, P. S. Talanta 2006, 70, 257-266; (c) Vocanson, F.; Seigle-Ferrand, P.; Lamartine, R.; Fort, A.; Coleman, A. W.; Shahgaldian, P.; Mugnier, J.; Zerroukki, A. J. Mater. Sci. 2003, 13, 1596-1602. 
(10) (a) Gutsche, C. D.; Dhawan, B.; Levine, J. A.; Kwang, H. N.; Bauer, L. Tetrahedron 1983, 39, 409-426; (b) Blixt, J.; Detellier, C. J. Am. Chem. Soc. 1995, 117, 8536-8540; (c) Harada, T.; Rudziński, J. M.; Shinkai, S. J. Chem. Soc., Perkin Trans. 2 1992, 2109-2115; (d) Van Loon, J.-D.; Groenen, L. C.; Wijmenga, S. S.; Verboom, W.; Reinhoud, D. N. J. Am. Chem. Soc. 1991, 113, 2378-2384.

(11) Gutsche, C. D.; Dhawan, B.; No, K. H.; Muthukrishnan, R. J. Am. Chem. Soc. 1981, 103, 3782-3792.

(12) Bitter, I.; Grün, A.; Ágai, B.; Tôke, L. Tetrahedron 1995, 51, 7835-7840.

(13) (a) Iwamoto, K.; Araki, K.; Shinkai, S. J. Org. Chem. 1991, 56, 4955-4962; (b) Gutsche, C. D.; Dhawan, B.; Levine, J. A.; No, K. H.; Bauer, L. J. Tetrahedron 1983, 39, 409-426.

(14) Böhmer, V. Angew. Chem. 1995, 107, 785-818; (Angew. Chem., Int. Ed. 1995, $34,713-745)$.

(15) For example, see: O`Malley, S.; Alhashimy, N.; O`Mahony, J.; Kieran, A.; Pryce, M.; Nolan, K. Tetrahedron Lett. 2007, 48, 681-684.

(16) Grootenhuis, P. D.; Kollman, P. A.; Groenen, L. C.; Reinhoudt, D. N.; van Hummerl, G. J.; Ugozzoli, F.; Andreetti, G. D. J. Am. Chem. Soc. 1990, 112, 4165 .

(17) Fischer, C.; Gruber, T.; Seichter, W.; Weber, E. Acta Cryst. 2007, E63, o4572o4573.

(18) Fischer, C.; Gruber, T.; Seichter, W.; Schindler, D.; Weber, E. Acta Cryst. 2008, E64, o673.

(19) Bodenhausen, G.; Ruben, D. J. Chem. Phys. Lett. 1980, 69, 185-189.

(20) (a) Summer, M. F.; Marzilli, L. G.; Bax, A. J. Am. Chem. Soc. 1986, 108, 42854294; (b) Bax, A.; Summer, M. F. J. Am. Chem. Soc. 1986, 108, 2093-2094; (c) Bax, A.; Aszalos, A.; Dinya, Z.; Sudo, K. J. Am. Chem. Soc. 1986, 108, 80568063.

(21) (a) Pons, M.; Millet, O. Progr. Nucl. Magn. Res. Spectr. 2001, 28, 267-324; (b) Perrin, C. L.; Dwyer, T. Chem. Rev. 1990, 90, 935-967.

(22) Halgren, T. A., J. Comput. Chem. 1999, 20, 730-748.

(23) Nishio, M., CrystEngComm 2004, 6, 130-158. 
(24) Smallwood, I. M. In Handbook of Organic Solvent Properties; Arnold: London, 1996.

(25) G. M. Sheldrick, SHELXL-97: Program for Crystal Structure Refinement; University of Göttingen, Germany, 1990.

(26) G. M. Sheldrick, SHELXL-97: Program for Crystal Structure Refinement; University of Göttingen, Germany, 1990. 
Table 1. (a) Rate constants $k_{\text {chem }}$ and (b) free activation energies $\Delta G^{\#}$ of conformational interconversion according to the methine protons $H$-1 of compounds 2-5 and for comparision of compound 1, calculated by the 2D-EXSY method.

(a)

\begin{tabular}{|c|c|c|c|c|c|c|c|c|c|c|}
\hline \multirow[t]{3}{*}{ Interconversion } & \multicolumn{10}{|c|}{$k_{\text {chem }}\left(\mathrm{s}^{-1}\right)$} \\
\hline & \multicolumn{4}{|c|}{2} & \multicolumn{4}{|c|}{3} & \multirow{2}{*}{$\begin{array}{c}\mathbf{4} \\
\mathrm{CDCl}_{3}, \\
265 \mathrm{~K}\end{array}$} & \multirow{2}{*}{$\begin{array}{c}\mathbf{5} \\
\mathrm{CDCl}_{3}, \\
265 \mathrm{~K}\end{array}$} \\
\hline & $\begin{array}{c}\mathrm{CDCl}_{3} \text {, } \\
295 \mathrm{~K}\end{array}$ & $\begin{array}{c}\mathrm{CDCl}_{3}, \\
264 \mathrm{~K}\end{array}$ & $\begin{array}{l}\text { TCl-d }{ }_{2} \text {, } \\
295 \mathrm{~K}\end{array}$ & $\begin{array}{c}\text { TCl-d } \\
\text {, } \\
263 \mathrm{~K}\end{array}$ & $\begin{array}{c}\mathrm{CDCl}_{3} \text {, } \\
295 \mathrm{~K}\end{array}$ & $\begin{array}{c}\mathrm{CDCl}_{3} \text {, } \\
263 \mathrm{~K}\end{array}$ & $\begin{array}{l}\text { TCl-d }{ }_{2} \text {, } \\
295 \mathrm{~K}\end{array}$ & $\begin{array}{c}\text { TCl-d }{ }_{2}, \\
260 \mathrm{~K}\end{array}$ & & \\
\hline paco/cone & fast & 18.9 & fast & - & fast & 18.2 & fast & - & 15.9 & 19.5 \\
\hline paco/1,2-alt. & 52.0 & 0.82 & 14.4 & 0.7 & 14.5 & 0.5 & 7.3 & 0.4 & 0.5 & 1.3 \\
\hline
\end{tabular}

(b)

\begin{tabular}{|c|c|c|c|c|c|c|c|c|c|c|}
\hline Interconversion & & & & & $\Delta G^{\#}$ & $\mathrm{~mol}^{-1}$ & & & & \\
\hline & & & & & & & & & 4 & 5 \\
\hline paco/cone & - & 13.8 & - & - & - & 13.7 & - & - & 13.9 & 13.7 \\
\hline $\mathrm{paco} / 1,2$-alt. & 14.9 & 15.4 & 15.7 & 15.6 & 15.7 & 15.6 & 16.1 & 15.6 & 15.7 & 15.2 \\
\hline
\end{tabular}

${ }^{\mathrm{a}}$ Interconversion paco/1,2-alt. for 3 at coalescence in TCl-d 2 (360 K): $17.5 \mathrm{kcal} \mathrm{mol}^{-1}$.

${ }^{\mathrm{b}}$ Interconversion paco/1,2-alt. for unsubstituted compound 1 at coalescence in TCl-d 2 (363 K): $16.4 \mathrm{kcal} \mathrm{mol}^{-1}$. 
Table 2. Mole fractions of conformers for compounds 1-5 in solution.

\begin{tabular}{|c|c|c|c|c|c|c|c|c|c|c|c|c|c|c|}
\hline \multirow[t]{2}{*}{ conformer } & \multicolumn{4}{|c|}{1} & \multicolumn{4}{|c|}{2} & \multicolumn{4}{|c|}{3} & \multirow{2}{*}{$\begin{array}{c}4 \\
\mathrm{CDCl}_{3}, \\
264 \mathrm{~K}\end{array}$} & \multirow{2}{*}{$\begin{array}{c}5 \\
\\
\mathrm{CDCl}_{3}, \\
263 \mathrm{~K}\end{array}$} \\
\hline & $\begin{array}{c}\mathrm{CDCl}_{3}, \\
295 \mathrm{~K}\end{array}$ & $\begin{array}{c}\mathrm{CDCl}_{3}, \\
265 \mathrm{~K}\end{array}$ & $\begin{array}{c}\mathrm{TCl}_{-\mathrm{d}_{2}} \\
295 \mathrm{~K}\end{array}$ & $\begin{array}{l}\mathrm{TCl}-\mathrm{d}_{2}, \\
263 \mathrm{~K}\end{array}$ & $\begin{array}{c}\mathrm{CDCl}_{3}, \\
295 \mathrm{~K}\end{array}$ & $\begin{array}{c}\mathrm{CDCl}_{3} \\
261 \mathrm{~K}\end{array}$ & $\begin{array}{l}\mathrm{TCl}-\mathrm{d}_{2} \\
295 \mathrm{~K}\end{array}$ & $\begin{array}{l}\mathrm{TCl}-\mathrm{d}_{2} \\
260 \mathrm{~K}\end{array}$ & $\begin{array}{c}\mathrm{CDCl}_{3}, \\
295 \mathrm{~K}\end{array}$ & $\begin{array}{c}\mathrm{CDCl}_{3} \\
261 \mathrm{~K}\end{array}$ & $\begin{array}{l}\mathrm{TCl}-\mathrm{d}_{2} \\
295 \mathrm{~K}\end{array}$ & $\begin{array}{c}\mathrm{TCl}-\mathrm{d}_{2} \\
260 \mathrm{~K}\end{array}$ & & \\
\hline $\begin{array}{c}\text { paco } \\
\left(\mathbf{\Delta}, \mathbf{\Lambda}^{\prime}\right)\end{array}$ & 85 & 84 & 83 & 84 & 90 & 74 & 88 & 79 & 88 & 72 & 87 & 80 & 73 & 71 \\
\hline $\begin{array}{l}\text { cone } \\
(0)\end{array}$ & 7 & 9 & 6 & 6 & - & 17 & - & 10 & - & 17 & - & 10 & 16 & 15 \\
\hline $\begin{array}{c}\text { 1,2-alt. } \\
\text { (M) }\end{array}$ & 8 & 7 & 10 & 9 & 10 & 9 & 12 & 11 & 12 & 10 & 13 & 10 & 11 & 14 \\
\hline
\end{tabular}


Table 3. Relative energies (in kcal mol ${ }^{-1}$ ) for possible conformers of ethyl compound 2 obtained by the MMFF94 force field (SYBYL) and calculated populations.

\begin{tabular}{cccc}
\hline Conformation & $\begin{array}{c}\text { Relative energy } \\
\left(\mathrm{kcal} \mathrm{mol}^{-1}\right)\end{array}$ & $\begin{array}{c}\text { Relative to pacoD } \\
\left(\mathrm{kcal} \mathrm{mol}^{-1}\right)\end{array}$ & $\begin{array}{c}\text { Population } \\
(\%)\end{array}$ \\
\hline coneA & 225.76 & 0.01 & 47.13 \\
coneB & 233.15 & 7.40 & - \\
pacoA & 233.99 & 8.24 & - \\
pacoB & 227.77 & 2.02 & 1.58 \\
pacoC & 227.58 & 1.83 & 2.18 \\
pacoD & 225.75 & 0.00 & 47.93 \\
alt $2 A$ & 237.02 & 11.27 & - \\
alt $2 B$ & 233.00 & 7.25 & - \\
alt $2 C$ & 229.50 & 3.75 & 0.08 \\
alt3 & 227.98 & 2.23 & 1.11 \\
\hline
\end{tabular}


Table 4. Crystal data and selected details of the data collection and refinement calculations of compound $\mathbf{2} \cdot \mathrm{CHCl}_{3}$.

\begin{tabular}{|c|c|}
\hline Compound & $2 \cdot \mathrm{CHCl}_{3}$ \\
\hline Empirical formula & $\mathrm{C}_{51} \mathrm{H}_{69} \mathrm{Cl}_{3} \mathrm{O}_{4}$ \\
\hline Formula weight $\left(\mathrm{g} \mathrm{mol}^{-1}\right)$ & 852.4 \\
\hline Crystal system & orthorhombic \\
\hline Space group & $\mathrm{Pca}_{1}$ \\
\hline$a(\AA)$ & $23.9269(5)$ \\
\hline$b(\AA)$ & $16.8437(4)$ \\
\hline$c(\AA)$ & $12.6634(3)$ \\
\hline$\alpha\left({ }^{\circ}\right)$ & 90.00 \\
\hline$\beta\left(^{\circ}\right)$ & 90.00 \\
\hline$\gamma\left({ }^{\circ}\right)$ & 90.00 \\
\hline$V\left(\AA^{3}\right)$ & $5103.6(2)$ \\
\hline$Z$ & 4 \\
\hline$F(000)$ & 1832 \\
\hline$D_{\mathrm{c}}\left(\mathrm{Mg} \mathrm{m}^{-3}\right)$ & 1.109 \\
\hline$\mu\left(\mathrm{mm}^{-1}\right)$ & 0.219 \\
\hline \multicolumn{2}{|l|}{ Data collection } \\
\hline Temperature (K) & 93(2) \\
\hline No. of collected reflections & 57108 \\
\hline within the $\theta$-limit $\left(^{\circ}\right)$ & $1.21-28.10$ \\
\hline Index ranges $\pm h, \pm k, \pm l$ & $-31 / 31,-22 / 21,-16 / 10$ \\
\hline No. of unique reflections & 9944 \\
\hline$R_{\text {int }}$ & 0.0968 \\
\hline $\begin{array}{l}\text { Refinement calculations: full- } \\
\text { matrix least- squares on all } F^{2} \\
\text { values }\end{array}$ & \\
\hline Weighting expression $w^{a}$ & $\begin{array}{l}{\left[\sigma^{2}\left(F_{\mathrm{o}}^{2}\right)+(0.1788 P)^{2}+\right.} \\
4.6405 P]^{-1}\end{array}$ \\
\hline No. of refined parameters & 537 \\
\hline $\begin{array}{l}\text { No. of } \mathrm{F} \text { values used }[I>2 \sigma(I)] \\
\text { Final } R \text {-Indices }\end{array}$ & 8817 \\
\hline$R\left(=\Sigma|\Delta F| / \Sigma\left|F_{\mathrm{o}}\right|\right)$ & 0.0843 \\
\hline$w R$ on $F^{2}$ & 0.2405 \\
\hline$S\left(=\right.$ Goodness of fit on $\left.F^{2}\right)$ & 1.092 \\
\hline Final $\Delta \rho_{\max } / \Delta \rho_{\min }\left(\mathrm{e} \AA^{-3}\right)$ & 0.000 \\
\hline
\end{tabular}

${ }^{a} P=\left(F_{\mathrm{o}}^{2}+2 F_{\mathrm{c}}^{2}\right)$ 


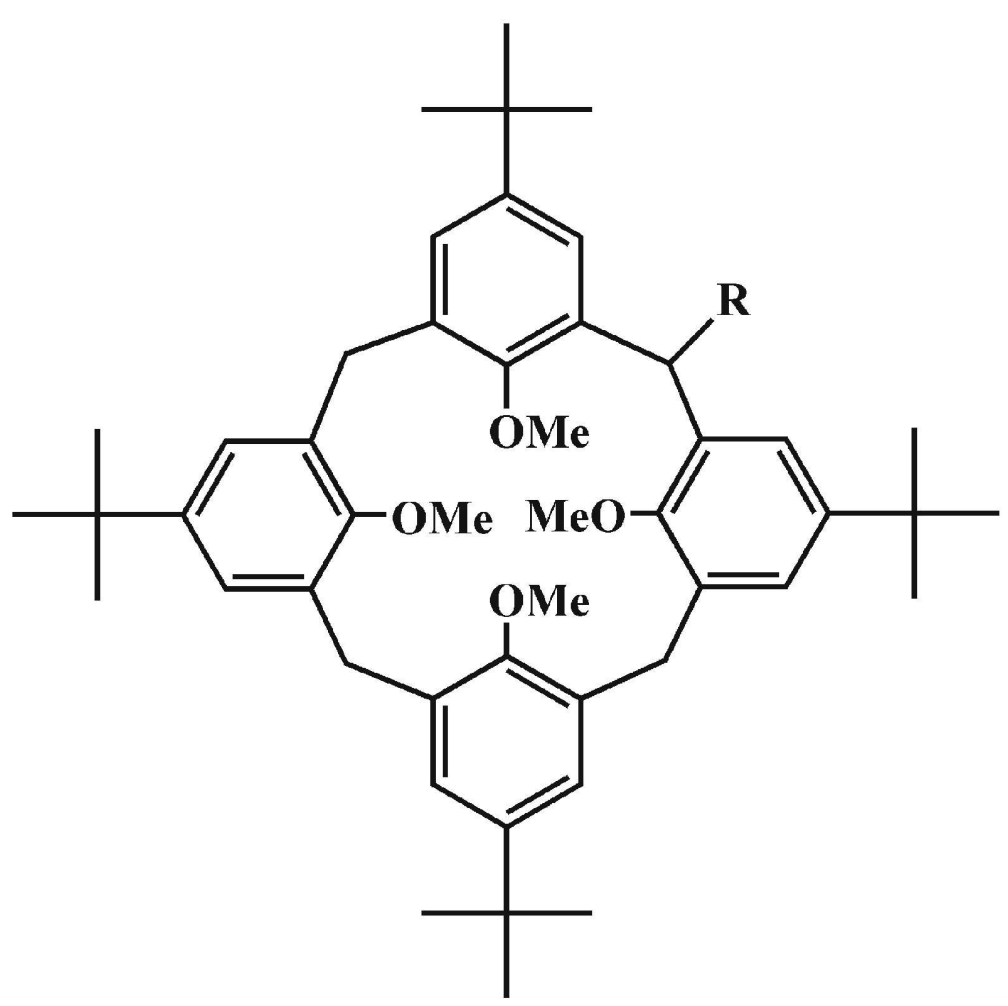

$1 \mathrm{R}=\mathrm{H}$

$2 \quad \mathrm{R}=\mathrm{C}_{2} \mathrm{H}_{5}$

$3 \mathrm{R}=n-\mathrm{C}_{3} \mathrm{H}_{7}$

$4 \mathrm{R}=n-\mathrm{C}_{5} \mathrm{H}_{11}$

$5 \mathrm{R}=n-\mathrm{C}_{10} \mathrm{H}_{21}$

$560 \times 877 \mathrm{~mm}(72 \times 72 \mathrm{DPI})$ 


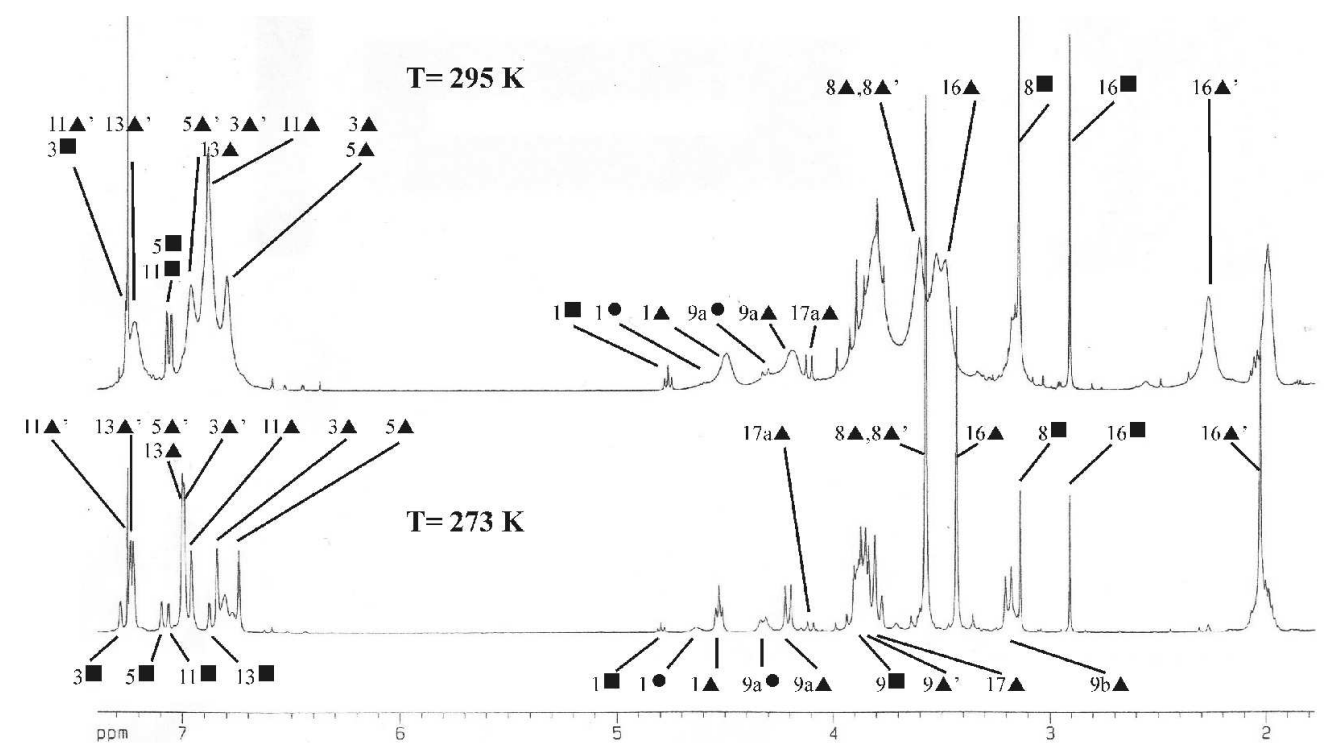

$1711 \times 974 \mathrm{~mm}(72 \times 72$ DPI $)$ 


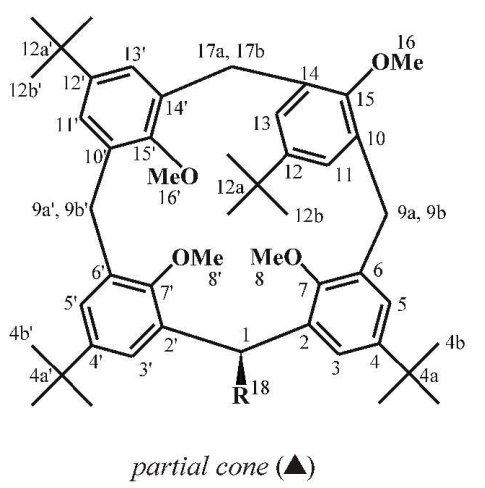

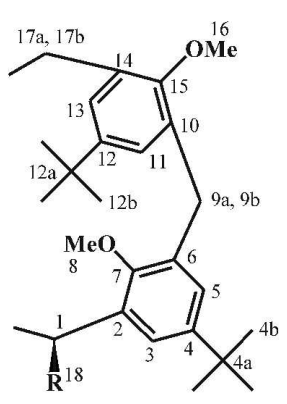

1,2-alternate (ם)

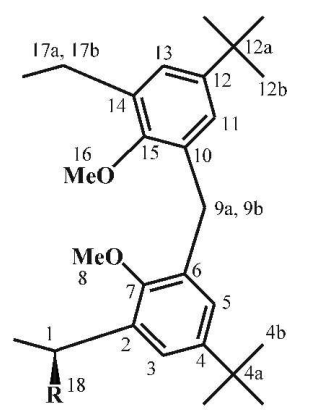

cone ()

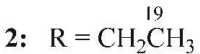

3: $\mathrm{R}=\mathrm{CH}_{2}{ }_{2} \mathrm{CH}_{2} \mathrm{CH}_{3}$

4: $\mathrm{R}=\mathrm{CH}_{2} \mathrm{CH}_{2}{ }_{20} \mathrm{CH}_{2}{ }^{21} \mathrm{CH}_{2} \mathrm{CH}_{3}$

5: $\mathrm{R}=\mathrm{CH}_{2} \mathrm{CH}_{2} \mathrm{CH}_{2} \mathrm{CH}_{2} \mathrm{CH}_{2} \mathrm{CH}_{2} \mathrm{CH}_{2}{ }_{2} \mathrm{CH}_{2}{ }^{26} \mathrm{CH}_{2}{ }_{2} \mathrm{CH}_{3}$ $1432 \times 751 \mathrm{~mm}(96 \times 96$ DPI) 

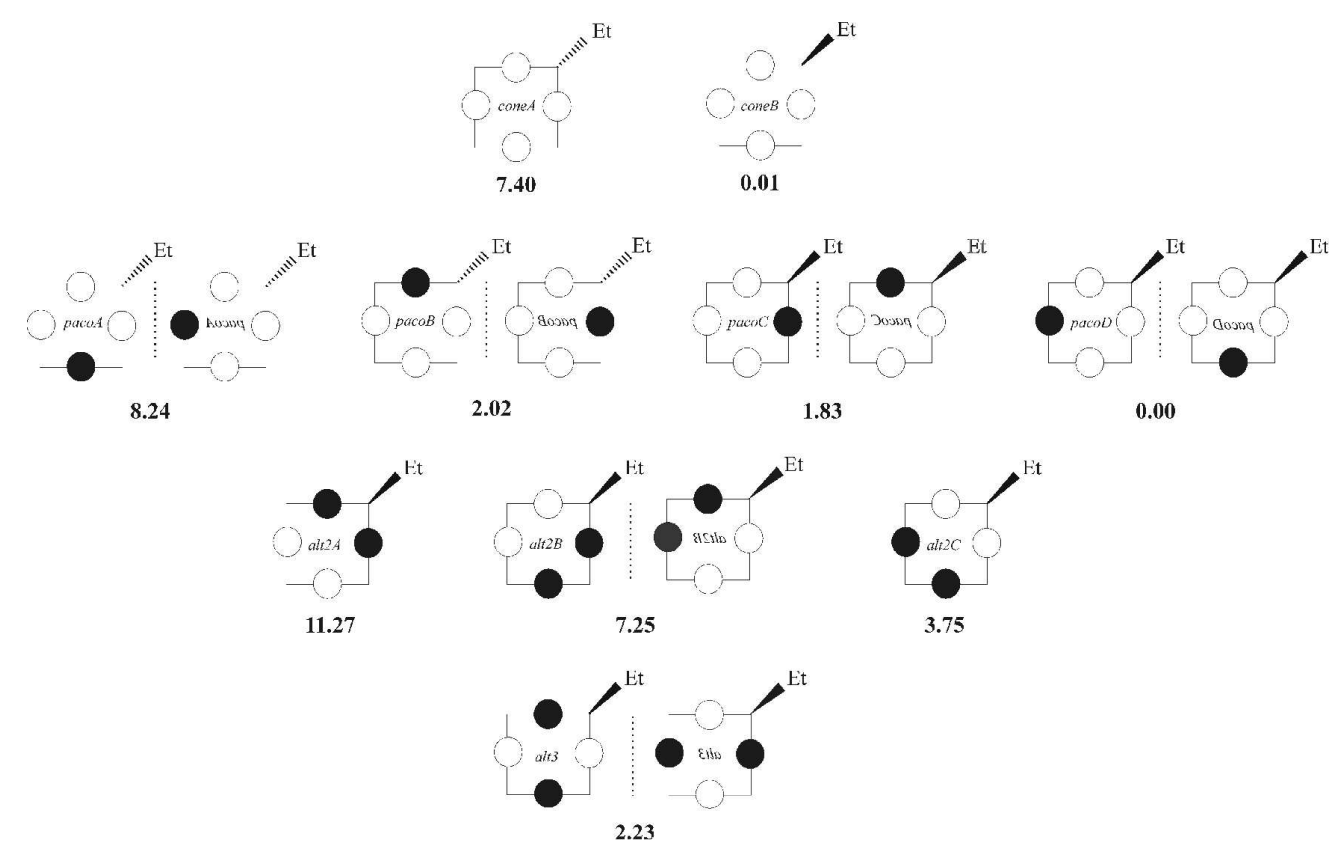

$1844 \times 1160 \mathrm{~mm}(72 \times 72 \mathrm{DPI})$

3

URL: http:/mc.manuscriptcentral.com/tandf/gsch Email: suprachem@mail.cm.utexas.edu 


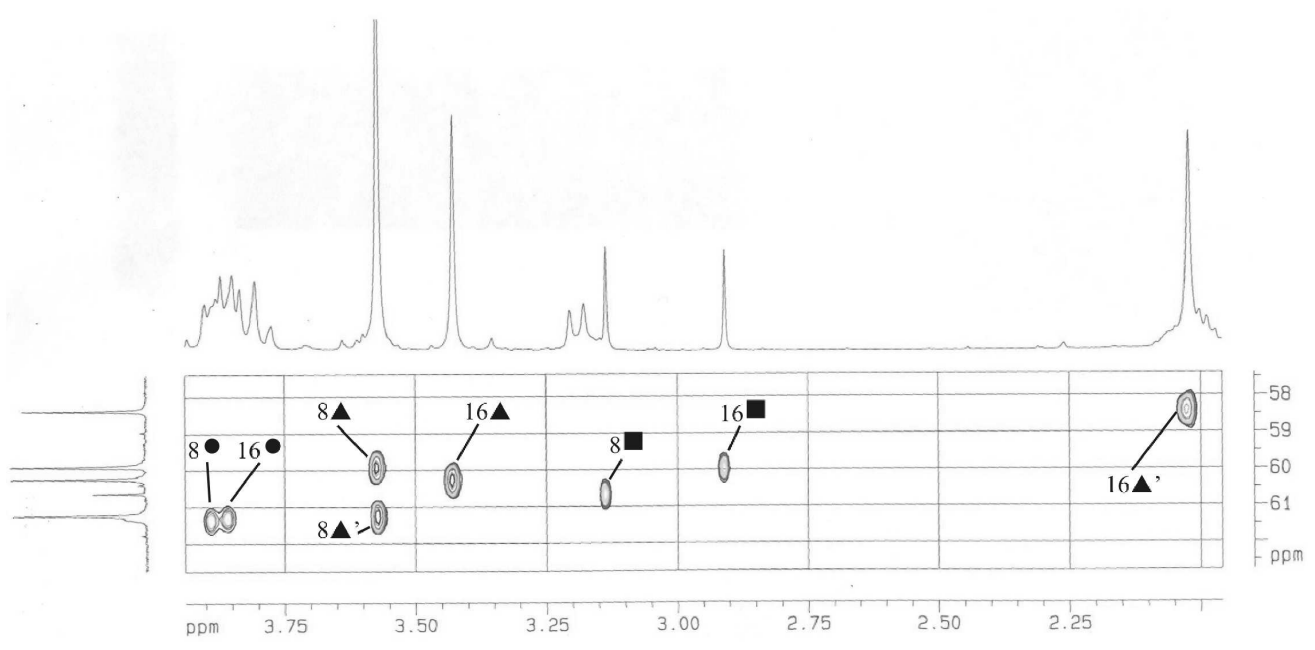

\section{$1603 \times 786 \mathrm{~mm}(72 \times 72 \mathrm{DPI})$}




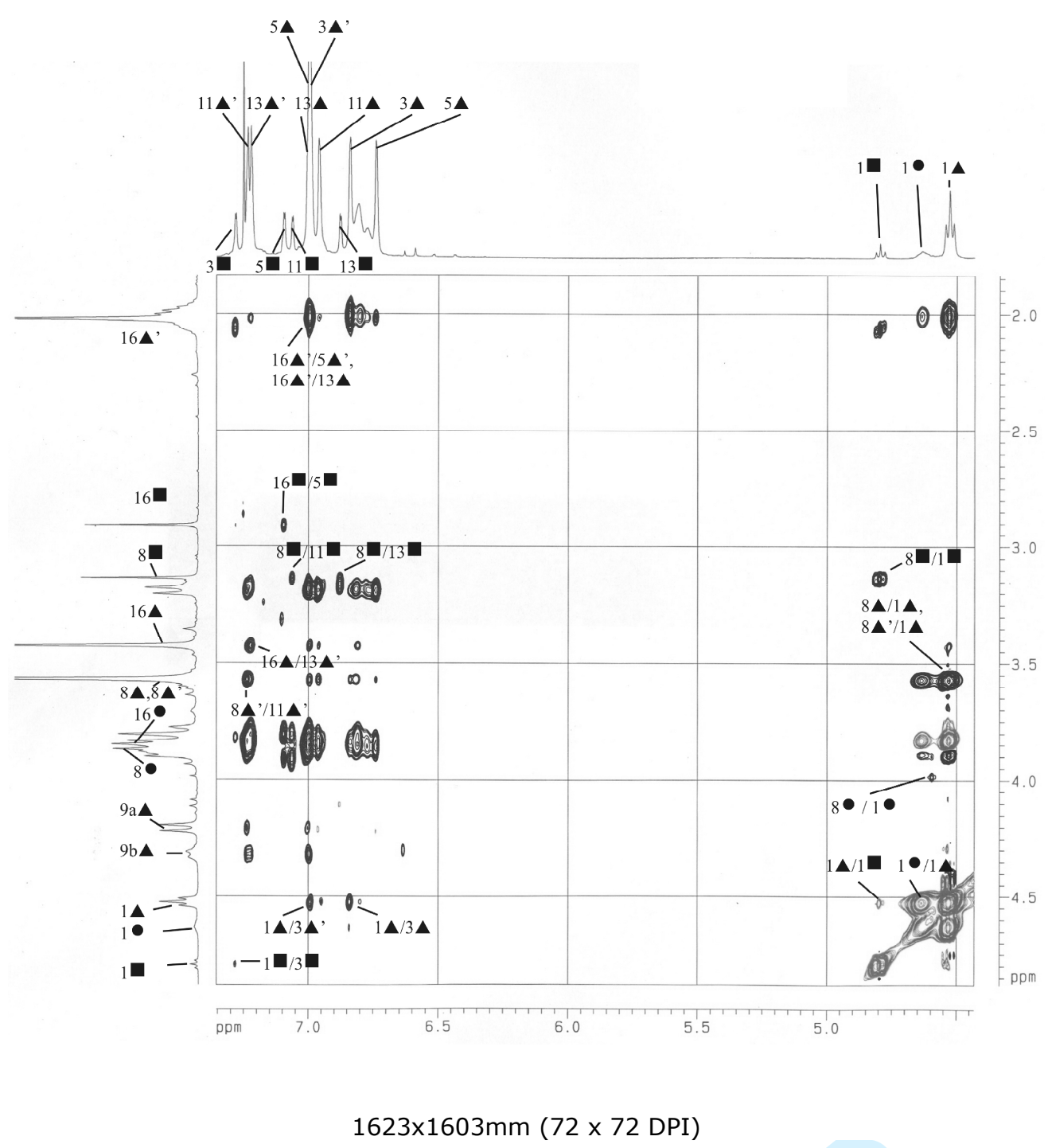




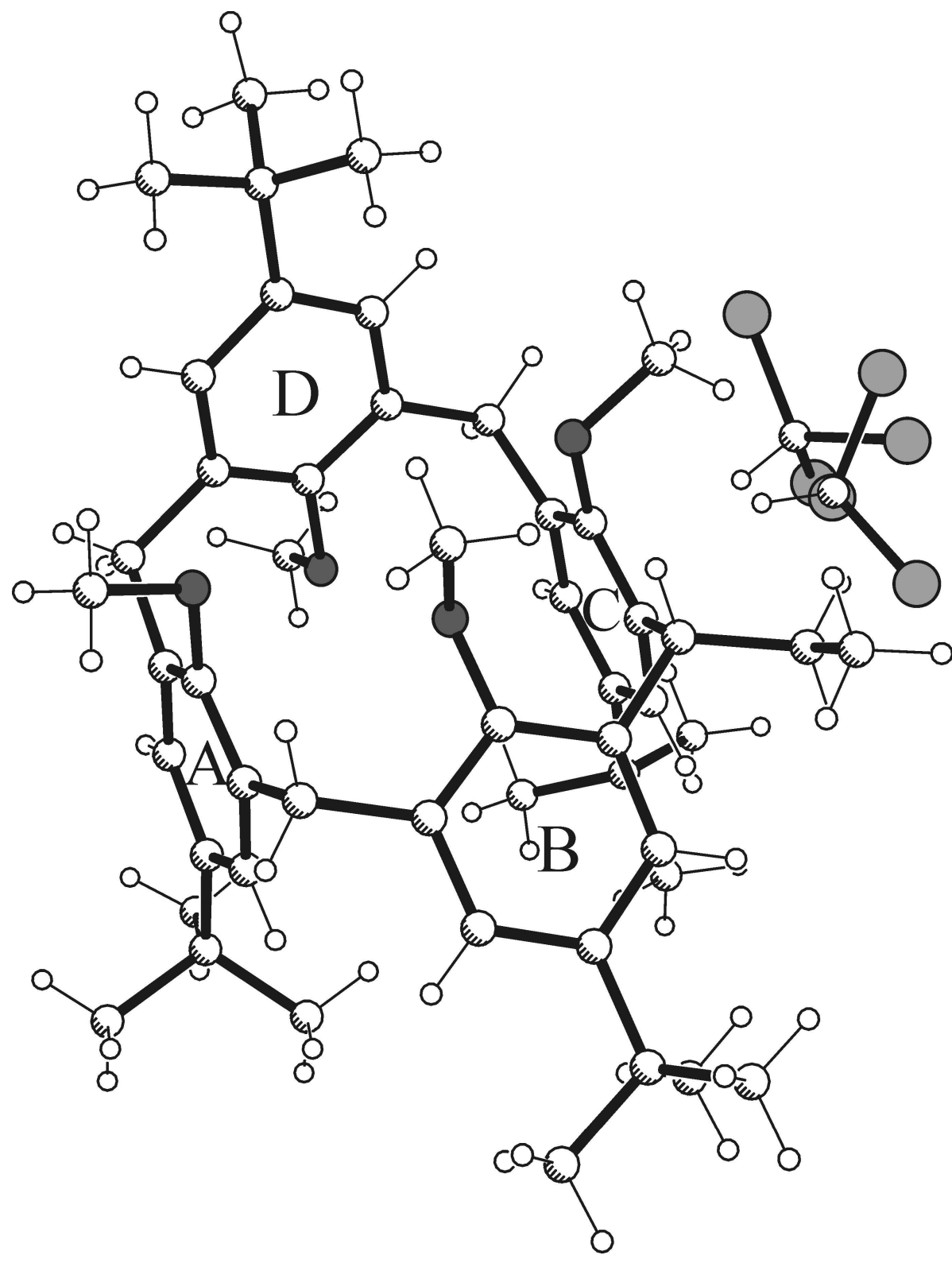

$981 \times 1292 \mathrm{~mm}(72 \times 72$ DPI $)$ 


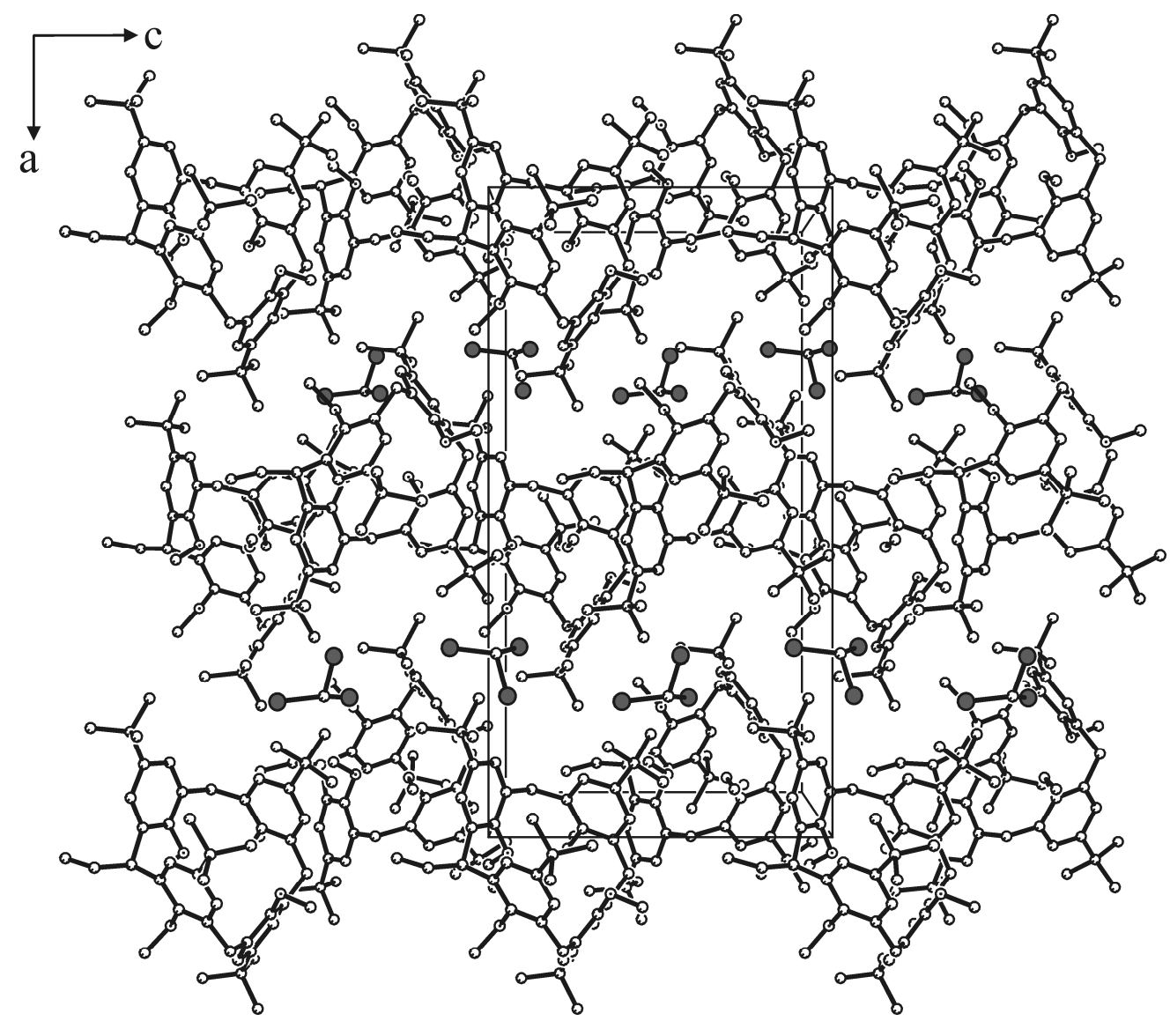

$1497 \times 1305 \mathrm{~mm}(72 \times 72 \mathrm{DPI})$ 

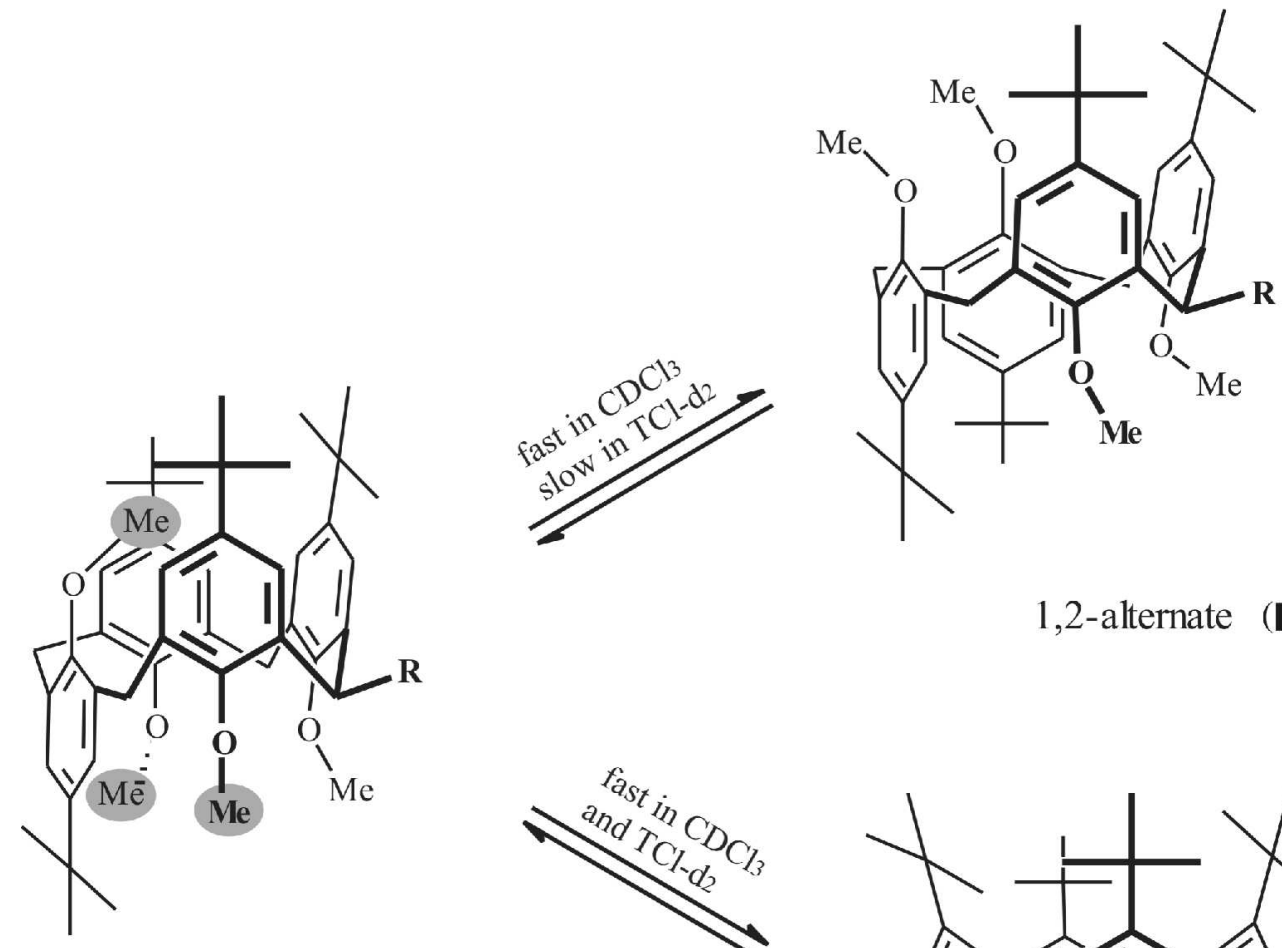

1,2-alternate

partial cone (A)
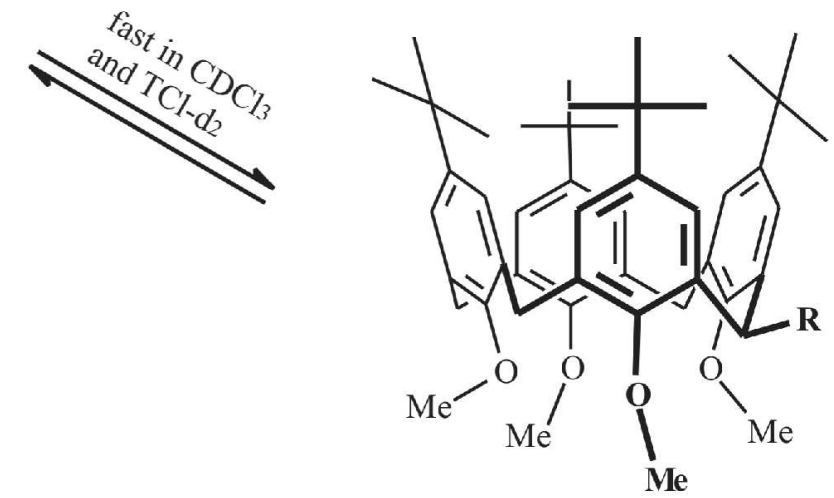

cone

$1108 \times 1189 \mathrm{~mm}(72 \times 72 \mathrm{DPI})$

URL: http:/mc.manuscriptcentral.com/tandf/gsch Email: suprachem@mail.cm.utexas.edu 


\section{Supplementary Materials}

Table S1a. $\quad{ }^{1} \mathrm{H}-\mathrm{NMR}$-spectrum of 2 in $\mathrm{CDCl}_{3}$ at $264 \mathrm{~K}$.

\begin{tabular}{|c|c|c|}
\hline $\operatorname{paco}\left(\boldsymbol{\Delta}, \mathbf{\Delta}^{\prime}\right)$ & 1,2-alternate $(0)$ & cone $(\mathrm{M})$ \\
\hline $7.24\left(\mathrm{~s}, \mathrm{br}, 1 \mathrm{H}, \mathrm{H}-11^{\prime}\right)$ & $7.28\left(\mathrm{~d},{ }^{4} \mathrm{~J}=2.0 \mathrm{~Hz}, 2 \mathrm{H}, \mathrm{H}-3\right)$ & $7.2(\mathrm{~s}, \mathrm{br}, \mathrm{H}-11)$ \\
\hline 7.22 (s, br, 1H, H-13') & $7.10(\mathrm{~d}, \mathrm{4} J=1.8 \mathrm{~Hz}, 2 \mathrm{H}, \mathrm{H}-5)$ & $6.81(\mathrm{~s}, \mathrm{br}, \mathrm{H}-13)$ \\
\hline $7.00\left(\mathrm{~s}, \mathrm{br}, 2 \mathrm{H}, \mathrm{H}-5^{\prime}\right)$ & $7.06\left(\mathrm{~d},{ }^{4} \mathrm{~J}=1.5 \mathrm{~Hz}, 2 \mathrm{H}, \mathrm{H}-11\right)$ & $6.81(\mathrm{~s}, \mathrm{br}, \mathrm{H}-3)$ \\
\hline $6.99(\mathrm{~s}, \mathrm{br}, 1 \mathrm{H}, \mathrm{H}-13)$ & $6.86\left(\mathrm{~d},{ }^{4} \mathrm{~J}=1.5 \mathrm{~Hz}, 2 \mathrm{H}, \mathrm{H}-13\right)$ & $6.76(\mathrm{~s}, \mathrm{br}, \mathrm{H}-5)$ \\
\hline \multicolumn{3}{|l|}{$6.98\left(\mathrm{~s}, \mathrm{br}, 1 \mathrm{H}, \mathrm{H}-3^{\prime}\right)$} \\
\hline \multicolumn{3}{|l|}{$6.96(\mathrm{~s}, \mathrm{br}, 1 \mathrm{H}, \mathrm{H}-11)$} \\
\hline \multicolumn{3}{|l|}{$6.84(\mathrm{~s}, \mathrm{br}, 1 \mathrm{H}, \mathrm{H}-3)$} \\
\hline \multicolumn{3}{|l|}{$6.74(\mathrm{~s}, \mathrm{br}, 1 \mathrm{H}, \mathrm{H}-5)$} \\
\hline $4.39\left(\mathrm{t}^{3}{ }^{3} \mathrm{~J}=8.0 \mathrm{~Hz}, \mathrm{H}-1\right)$ & $4.70\left(\mathrm{t},{ }^{3} \mathrm{~J}=8.2 \mathrm{~Hz}, 1 \mathrm{H}, \mathrm{H}-1\right)$ & $4.48(\mathrm{t}, \mathrm{br}, 1 \mathrm{H}, \mathrm{H}-1)$ \\
\hline $4.21\left(\mathrm{~d},{ }^{2} \mathrm{~J}=12.8 \mathrm{~Hz}, \mathrm{H}-9 \mathrm{a}\right)$ & $4.10\left(\mathrm{~d},{ }^{2} \mathrm{~J}=13.4 \mathrm{~Hz}, 1 \mathrm{H}, \mathrm{H}-17 \mathrm{a}\right)$ & $4.32(\mathrm{~d}, \mathrm{br}, \mathrm{H}-9 \mathrm{a})$ \\
\hline $3.90\left(\mathrm{~d},{ }^{2} \mathrm{~J}=15.4 \mathrm{~Hz}, \mathrm{H}-9 \mathrm{a}{ }^{\prime}\right)$ & $3.19(1 \mathrm{H}, \mathrm{H}-17 \mathrm{~b})$ & $3.18(\mathrm{H}-9 \mathrm{~b})$ \\
\hline $3.86\left(\mathrm{~d},{ }^{2} \mathrm{~J}=15.4 \mathrm{~Hz}, \mathrm{H}-17 \mathrm{a}\right)$ & $3.93(1 \mathrm{H}, \mathrm{H}-9 \mathrm{a})$ & $4.12(\mathrm{H}-17 \mathrm{a})$ \\
\hline $3.80\left(\mathrm{~d},{ }^{2} \mathrm{~J} \sim 15 \mathrm{~Hz}, \mathrm{H}-9 \mathrm{~b}\right)$ & $3.78(1 \mathrm{H}, \mathrm{H}-9 \mathrm{~b})$ & $3.17(\mathrm{H}-17 \mathrm{~b})$ \\
\hline \multicolumn{3}{|l|}{$3.79\left(\mathrm{~d},{ }^{2} \mathrm{~J}=15.3 \mathrm{~Hz}, \mathrm{H}-17 \mathrm{~b}\right)$} \\
\hline \multicolumn{3}{|l|}{$3.19\left(\mathrm{~d},{ }^{2} \mathrm{~J}=13.0 \mathrm{~Hz}, \mathrm{H}-9 \mathrm{~b}\right)$} \\
\hline $3.57\left(\mathrm{~s}, 3 \mathrm{H}, \mathrm{H}-8^{\prime}\right)$ & $3.14(\mathrm{~s}, 6 \mathrm{H}, \mathrm{H}-8)$ & $3.87(\mathrm{H}-8)$ \\
\hline $3.56(\mathrm{~s}, 3 \mathrm{H}, \mathrm{H}-8)$ & $2.90(\mathrm{~s}, 6 \mathrm{H}, \mathrm{H}-16)$ & $3.85(\mathrm{H}-16)$ \\
\hline \multicolumn{3}{|l|}{$3.42(\mathrm{~s}, 3 \mathrm{H}, \mathrm{H}-16)$} \\
\hline \multicolumn{3}{|l|}{2.03 (s, 3H, H-16') } \\
\hline $1.39\left(\mathrm{~s}, 9 \mathrm{H}, \mathrm{H}-12 \mathrm{~b}^{\prime}\right)$ & $1.29(\mathrm{~s}, 18 \mathrm{H}, \mathrm{H}-4 \mathrm{~b})$ & not discriminable \\
\hline 1.23 (s, 9H, H-12b) & $1.25(\mathrm{~s}, 18 \mathrm{H}, \mathrm{H}-12 \mathrm{~b})$ & \\
\hline \multicolumn{3}{|l|}{$1.22(\mathrm{~s}, 9 \mathrm{H}, \mathrm{H}-4 \mathrm{~b}$ ') } \\
\hline \multicolumn{3}{|l|}{1.09 (s, 9H, H-4b) } \\
\hline $2.06(\mathrm{~m}, 2 \mathrm{H}, \mathrm{H}-18)$ & $2.09(\mathrm{~m}, 2 \mathrm{H}, \mathrm{H}-18)$ & $2.06(\mathrm{H}-18)$ \\
\hline $1.05\left(\mathrm{t}, 3 \mathrm{H},{ }^{3} \mathrm{~J}=7.2 \mathrm{~Hz}, \mathrm{H}-19\right)$ & $0.99(\mathrm{t}, 3 \mathrm{H}, \mathrm{H}-19)$ & $1.05(\mathrm{H}-19)$ \\
\hline
\end{tabular}


Table S1b. ${ }^{13} \mathrm{C}-\mathrm{NMR}-$ spectrum of 2 in $\mathrm{CDCl}_{3}$ at $264 \mathrm{~K}$.

\begin{tabular}{|c|c|c|c|}
\hline & $\operatorname{paco}\left(\boldsymbol{\Delta}, \mathbf{\Lambda}^{\prime}\right)$ & 1,2-alternate $(0)$ & $\operatorname{cone}(\mathrm{M})$ \\
\hline $154.84\left(\mathrm{C}_{\mathrm{i}}-15\right)$ & $155.11\left(\mathrm{C}_{\mathrm{i}}-7^{\prime}\right)$ & $154.64\left(\mathrm{C}_{\mathrm{i}}-15\right)$ & $154.9\left(\mathrm{br}, \mathrm{C}_{\mathrm{i}}-15\right)$ \\
\hline $154.59\left(\mathrm{C}_{\mathrm{i}}-7\right)$ & $154.64\left(\mathrm{C}_{\mathrm{i}}-15^{\prime}\right)$ & $153.79\left(\mathrm{C}_{\mathrm{i}}-7\right)$ & \\
\hline $144.91\left(\mathrm{C}_{\mathrm{i}}-12\right)$ & $145.01\left(\mathrm{C}_{\mathrm{i}}-4^{\prime}\right)$ & $144.45\left(\mathrm{C}_{\mathrm{i}}-12\right)$ & $144.3\left(\mathrm{br}, \mathrm{C}_{\mathrm{i}}-4,12\right)$ \\
\hline $144.53\left(\mathrm{C}_{\mathrm{i}}-4\right)$ & $143.22\left(\mathrm{C}_{\mathrm{i}}-12^{\prime}\right)$ & $144.00\left(C_{i}-4\right)$ & \\
\hline $136.84\left(\mathrm{C}_{\mathrm{i}}-2\right)$ & $137.84\left(\mathrm{C}_{\mathrm{i}}-2^{\prime}\right)$ & $137.97\left(\mathrm{C}_{\mathrm{i}}-2\right)$ & $137.1\left(\mathrm{br}, \mathrm{C}_{\mathrm{i}}-2\right)$ \\
\hline $135.24\left(\mathrm{C}_{\mathrm{i}}-10\right)$ & $133.13\left(\mathrm{C}_{\mathrm{i}}-10^{\prime}\right)$ & $135.11\left(\mathrm{C}_{\mathrm{i}}-14\right)$ & $133.9\left(\mathrm{br}, \mathrm{C}_{\mathrm{i}}-10\right)$ \\
\hline $133.34\left(\mathrm{C}_{\mathrm{i}}-6\right)$ & $133.13\left(\mathrm{C}_{\mathrm{i}}-14^{\prime}\right)$ & $132.68\left(\mathrm{C}_{\mathrm{i}}-10\right)$ & $133.8\left(\mathrm{br}, \mathrm{C}_{\mathrm{i}}-14\right)$ \\
\hline $132.77\left(\mathrm{C}_{\mathrm{i}}-14\right)$ & $132.74\left(\mathrm{C}_{\mathrm{i}}-6^{\prime}\right)$ & $132.60\left(C_{i}-6\right)$ & $133.5\left(\mathrm{br}, \mathrm{C}_{\mathrm{i}}-6\right)$ \\
\hline $125.62(\mathrm{CH}, \mathrm{C}-13)$ & $126.39\left(\mathrm{CH}, \mathrm{C}-13^{\prime}\right)$ & $126.15(\mathrm{CH}, \mathrm{C}-5)$ & $126.3(\mathrm{br}, \mathrm{C}-11)$ \\
\hline $125.10(\mathrm{CH}, \mathrm{C}-11)$ & $126.30\left(\mathrm{CH}, \mathrm{C}-11^{\prime}\right)$ & $125.86(\mathrm{CH}, \mathrm{C}-11)$ & 124.4 (br, C-13) \\
\hline $124.53(\mathrm{CH}, \mathrm{C}-5)$ & $125.41\left(\mathrm{CH}, \mathrm{C}-5^{\prime}\right)$ & $125.31(\mathrm{CH}, \mathrm{C}-13)$ & $124.0(\mathrm{br}, \mathrm{C}-5)$ \\
\hline $121.84(\mathrm{CH}, \mathrm{C}-3)$ & $121.85\left(\mathrm{CH}, \mathrm{C}-3^{\prime}\right)$ & $122.04(\mathrm{CH}, \mathrm{C}-3)$ & $121.4(\mathrm{br}, \mathrm{C}-3)$ \\
\hline $60.24\left(\mathrm{CH}_{3}, \mathrm{C}-16\right)$ & $59.90\left(\mathrm{CH}_{3}, \mathrm{C}-8{ }^{\prime}\right)$ & $60.64\left(\mathrm{CH}_{3}, \mathrm{C}-8\right)$ & $61.23\left(\mathrm{CH}_{3}, \mathrm{C}-8\right)$ \\
\hline $61.21\left(\mathrm{CH}_{3}, \mathrm{C}-8\right)$ & $58.36\left(\mathrm{CH}_{3}, \mathrm{C}-16{ }^{\prime}\right)$ & $59.91\left(\mathrm{CH}_{3}, \mathrm{C}-16\right)$ & $61.20\left(\mathrm{CH}_{3}, \mathrm{C}-16\right)$ \\
\hline $36.89(\mathrm{CH}, \mathrm{C}-1)$ & & $36.27(\mathrm{CH}, \mathrm{C}-1)$ & $37.39(\mathrm{CH}, \mathrm{C}-1)$ \\
\hline $36.86\left(\mathrm{CH}_{2}, \mathrm{C}-17\right)$ & $37.55\left(\mathrm{CH}_{2}, \mathrm{C}-9^{\prime}\right)$ & $36.86\left(\mathrm{CH}_{2}, \mathrm{C}-9\right)$ & $31.0\left(\mathrm{CH}_{2}, \mathrm{C}-9\right)$ \\
\hline $31.00\left(\mathrm{CH}_{2}, \mathrm{C}-9\right)$ & & $30.11\left(\mathrm{CH}_{2}, \mathrm{C}-17\right)$ & $30.9\left(\mathrm{CH}_{2}, \mathrm{C}-17\right)$ \\
\hline $26.87\left(\mathrm{CH}_{2}, \mathrm{C}-18\right)$ & & $27.72\left(\mathrm{CH}_{2}, \mathrm{C}-18\right)$ & $26.87\left(\mathrm{CH}_{2}, \mathrm{C}-18\right)$ \\
\hline $13.48\left(\mathrm{CH}_{3}, \mathrm{C}-19\right)$ & & $13.39\left(\mathrm{CH}_{3}, \mathrm{C}-19\right)$ & $13.50\left(\mathrm{CH}_{3}, \mathrm{C}-19\right)$ \\
\hline $3.88(\mathrm{C}, \mathrm{C}-4 \mathrm{a})$ & 34.02 (C,C-4a') & $34.04(\mathrm{C}, \mathrm{C}-4 \mathrm{a})$ & 33.8 (br, C, C-4a, 12a) \\
\hline $33.88(\mathrm{C}, \mathrm{C}-12 \mathrm{a})$ & 33.88 (C,C-12a') & $33.92(\mathrm{C}, \mathrm{C}-12 \mathrm{a})$ & \\
\hline $31.41\left(\mathrm{CH}_{3}, \mathrm{C}-12 \mathrm{~b}\right)$ & $31.53\left(\mathrm{CH}_{3}, \mathrm{C}-12 \mathrm{~b}^{\prime}\right)$ & $31.47\left(\mathrm{CH}_{3}, \mathrm{C}-4 \mathrm{~b}\right)$ & \\
\hline $31.28\left(\mathrm{CH}_{3}, \mathrm{C}-4 \mathrm{~b}\right)$ & $31.36\left(\mathrm{CH}_{3}, \mathrm{C}-4 \mathrm{~b}^{\prime}\right)$ & $31.36\left(\mathrm{CH}_{3}, \mathrm{C}-12 \mathrm{~b}\right)$ & \\
\hline
\end{tabular}


Table S1c. $\quad{ }^{1} \mathrm{H}-\mathrm{NMR}-$ spectrum of 2 in $\mathrm{TCl}-\mathrm{d}_{2}$ at $263 \mathrm{~K}$.

\begin{tabular}{|c|c|c|}
\hline $\operatorname{paco}\left(\boldsymbol{\Lambda}, \mathbf{\Lambda}^{\prime}\right)$ & 1,2-alternate $(0)$ & cone $(\mathrm{M})$ \\
\hline 7.06 (s, br, 1H, H-11') & $7.06(\mathrm{~s}, \mathrm{br}, \mathrm{H}-3)$ & $7.09(\mathrm{H}-11)$ \\
\hline 7.05 (s, br, 1H, H-13') & $6.92(\mathrm{~s}, \mathrm{br}, \mathrm{H}-5)$ & 6.6-6.7 (H-3, 5, \\
\hline $6.92\left(\mathrm{~s}, \mathrm{br}, 1 \mathrm{H}, \mathrm{H}-3{ }^{\prime}\right)$ & $6.90(\mathrm{~d}, \mathrm{br}, 2 \mathrm{H}, \mathrm{H}-11)$ & 13) \\
\hline $6.87(\mathrm{~s}, \mathrm{br}, 1 \mathrm{H}, \mathrm{H}-11)$ & $6.74(\mathrm{~d}, \mathrm{br}, 2 \mathrm{H}, \mathrm{H}-13)$ & \\
\hline \multicolumn{3}{|l|}{$6.83\left(\mathrm{~s}, \mathrm{br}, 2 \mathrm{H}, \mathrm{H}-13,5^{\prime}\right)$} \\
\hline \multicolumn{3}{|l|}{$6.59(\mathrm{~s}, \mathrm{br}, 1 \mathrm{H}, \mathrm{H}-3)$} \\
\hline \multicolumn{3}{|l|}{$6.52(\mathrm{~s}, \mathrm{br}, 1 \mathrm{H}, \mathrm{H}-5)$} \\
\hline $4.25\left(\mathrm{t},{ }^{3} \mathrm{~J}=7.8 \mathrm{~Hz}, \mathrm{H}-1\right)$ & $4.43\left(\mathrm{t},{ }^{3} \mathrm{~J}=7.8 \mathrm{~Hz}, 1 \mathrm{H}, \mathrm{H}-1\right)$ & $4.3(\mathrm{t}, \mathrm{br}, \mathrm{H}-1)$ \\
\hline $4.06\left(\mathrm{~d},{ }^{2} \mathrm{~J}=12.5 \mathrm{~Hz}, \mathrm{H}-9 \mathrm{a}\right)$ & $3.96(\mathrm{~d}, 1 \mathrm{H}, \mathrm{H}-17 \mathrm{a})$ & 4.14 (br, H-9a) \\
\hline $3.75(\mathrm{~d}, \mathrm{H}-17 \mathrm{a})$ & $3.73(\mathrm{~d}, 2 \mathrm{H}, \mathrm{H}-9 \mathrm{a})$ & 3.03 (br, H-9b) \\
\hline $3.69(\mathrm{~d}, \mathrm{H}-17 \mathrm{~b})$ & $3.64(\mathrm{~d}, 2 \mathrm{H}, \mathrm{H}-9 \mathrm{~b})$ & $4.00(\mathrm{br}, \mathrm{H}-17 \mathrm{a})$ \\
\hline $3.09\left(\mathrm{~d},{ }^{2} \mathrm{~J}=12.4 \mathrm{~Hz}, \mathrm{H}-9 \mathrm{~b}\right)$ & $3.02(\mathrm{~d}, 1 \mathrm{H}, \mathrm{H}-17 \mathrm{~b})$ & 3.05 (br, H-17b) \\
\hline \multicolumn{3}{|l|}{3.73 (d, ${ }^{2} \mathrm{~J} \sim 10 \mathrm{~Hz}, \mathrm{H}-9 \mathrm{a}$ ') } \\
\hline \multicolumn{3}{|l|}{$3.70\left(\mathrm{~d}^{2}{ }^{2} \mathrm{~J} \sim 10 \mathrm{~Hz}, \mathrm{H}-9 \mathrm{~b}\right)$} \\
\hline $3.48(\mathrm{~s}, 3 \mathrm{H}, \mathrm{H}-8)$ & $2.96(\mathrm{~s}, 6 \mathrm{H}, \mathrm{H}-8)$ & $3.74(\mathrm{H}-8)$ \\
\hline $3.39\left(\mathrm{~s}, 3 \mathrm{H}, \mathrm{H}-8^{\prime}\right)$ & $2.76(\mathrm{~s}, 6 \mathrm{H}, \mathrm{H}-16)$ & $3.71(\mathrm{H}-16)$ \\
\hline \multicolumn{3}{|l|}{$3.25(\mathrm{~s}, 3 \mathrm{H}, \mathrm{H}-16)$} \\
\hline \multicolumn{3}{|l|}{$1.60\left(\mathrm{~s}, 3 \mathrm{H}, \mathrm{H}-16^{\prime}\right)$} \\
\hline $1.25\left(\mathrm{~s}, 9 \mathrm{H}, \mathrm{H}-12 \mathrm{~b}^{\prime}\right)$ & $1.15(\mathrm{~s}, 18 \mathrm{H}, \mathrm{H}-4 \mathrm{~b})$ & $1.29(\mathrm{H}-12 \mathrm{~b})$ \\
\hline $1.12\left(\mathrm{~s}, 18 \mathrm{H}, \mathrm{H}-4 \mathrm{~b}^{\prime}, 12 \mathrm{~b}^{\prime}\right)$ & $1.12(\mathrm{~s}, 18 \mathrm{H}, \mathrm{H}-12 \mathrm{~b})$ & $1.11(\mathrm{H}-4 \mathrm{~b})$ \\
\hline \multicolumn{3}{|l|}{$0.90(\mathrm{~s}, 9 \mathrm{H}, \mathrm{H}-4 \mathrm{~b})$} \\
\hline $1.96(\mathrm{~m}, 1 \mathrm{H}, \mathrm{H}-18 \mathrm{a})$ & $1.93(\mathrm{~m}, 1 \mathrm{H}, \mathrm{H}-18)$ & not discriminable \\
\hline $1.89(\mathrm{~m}, 1 \mathrm{H}, \mathrm{H}-18 \mathrm{~b})$ & $0.86(\mathrm{t}, 3 \mathrm{H}, \mathrm{H}-19)$ & \\
\hline $0.90(\mathrm{t}, 3 \mathrm{H}, \mathrm{H}-19)$ & & \\
\hline
\end{tabular}


Table S1d. $\quad{ }^{13} \mathrm{C}-\mathrm{NMR}-$ spectrum of 2 in $\mathrm{TCl}-\mathrm{d}_{2}$ at $263 \mathrm{~K}$.

\begin{tabular}{|c|c|c|c|}
\hline \multicolumn{2}{|c|}{$\operatorname{paco}\left(\boldsymbol{\Delta}, \mathbf{\Delta}^{\prime}\right)$} & \multirow{2}{*}{$\begin{array}{c}\text { 1,2-alternate }(0) \\
154.04\left(\mathrm{C}_{\mathrm{i}}-7\right)\end{array}$} & \multirow[t]{2}{*}{ cone $(\mathrm{M})$} \\
\hline $154.75\left(\mathrm{C}_{\mathrm{i}}-7\right)$ & $155.47\left(\mathrm{C}_{\mathrm{i}} \mathbf{7}^{\prime}\right)$ & & \\
\hline $155.10\left(\mathrm{C}_{\mathrm{i}}-15\right)$ & $154.84\left(\mathrm{C}_{\mathrm{i}}-15^{\prime}\right)$ & $154.75\left(\mathrm{C}_{\mathrm{i}}-15\right)$ & \\
\hline $144.60\left(\mathrm{C}_{\mathrm{i}}-4\right)$ & $145.32\left(\mathrm{C}_{\mathrm{i}}-4^{\prime}\right)$ & $144.27\left(\mathrm{C}_{\mathrm{i}}-4\right)$ & \\
\hline $145.28\left(\mathrm{C}_{\mathrm{i}}-12\right)$ & $143.55\left(\mathrm{C}_{\mathrm{i}}-12^{\prime}\right)$ & $145.32\left(\mathrm{C}_{\mathrm{i}}-12\right)$ & \\
\hline $137.00\left(\mathrm{C}_{\mathrm{i}}-2\right)$ & $138.39\left(\mathrm{C}_{\mathrm{i}}-2^{\prime}\right)$ & $138.30\left(\mathrm{C}_{\mathrm{i}}-2\right)$ & \\
\hline $136.05\left(\mathrm{C}_{\mathrm{i}}-10\right)$ & $133.38\left(\mathrm{C}_{\mathrm{i}}-10^{\prime}\right)$ & $132.95\left(\mathrm{C}_{\mathrm{i}}-10\right)$ & \\
\hline $133.10\left(\mathrm{C}_{\mathrm{i}}-14\right)$ & $133.38\left(\mathrm{C}_{\mathrm{i}}-14^{\prime}\right)$ & $135.33\left(\mathrm{C}_{\mathrm{i}}-14\right)$ & \\
\hline $133.09\left(\mathrm{C}_{\mathrm{i}}-6\right)$ & $133.10\left(\mathrm{C}_{\mathrm{i}}-6^{\prime}\right)$ & $132.83\left(\mathrm{C}_{\mathrm{i}}-6\right)$ & \\
\hline $125.93(\mathrm{CH}, \mathrm{C}-13)$ & $126.68\left(\mathrm{CH}, \mathrm{C}-11^{\prime}\right)$ & $126.43(\mathrm{CH}, \mathrm{C}-5)$ & $126.7(\mathrm{CH}, \mathrm{C}-11)$ \\
\hline $125.61(\mathrm{CH}, \mathrm{C}-11)$ & $(\mathrm{CH}, \mathrm{C}-13$ ') & $126.28(\mathrm{CH}, \mathrm{C}-11)$ & $124.9(\mathrm{CH}, \mathrm{C}-13)$ \\
\hline 124.87 (CH,C-5) & $125.60\left(\mathrm{CH}, \mathrm{C}-5^{\prime}\right)$ & $125.74(\mathrm{CH}, \mathrm{C}-13)$ & $124.4(\mathrm{CH}, \mathrm{C}-5)$ \\
\hline 122.47 (CH,C-3) & $122.03\left(\mathrm{CH}, \mathrm{C}-3^{\prime}\right)$ & $122.47(\mathrm{CH}, \mathrm{C}-3)$ & $121.9(\mathrm{CH}, \mathrm{C}-9)$ \\
\hline $61.84\left(\mathrm{CH}_{3}, \mathrm{C}-8\right)$ & $60.29\left(\mathrm{CH}_{3}, \mathrm{C}-8^{\prime}\right)$ & $60.85\left(\mathrm{CH}_{3}, \mathrm{C}-8\right)$ & $61.5\left(b r, \mathrm{CH}_{3}\right.$ \\
\hline $60.53\left(\mathrm{CH}_{3}, \mathrm{C}-16\right)$ & $58.77\left(\mathrm{CH}_{3}, \mathrm{C}-16^{\prime}\right)$ & $60.29\left(\mathrm{CH}_{3}, \mathrm{C}-16\right)$ & C-8;16) \\
\hline $37.89(\mathrm{CH}, \mathrm{C}-1)$ & & $36.73(\mathrm{CH}, \mathrm{C}-1)$ & +2 \\
\hline $31.36\left(\mathrm{CH}_{2}, \mathrm{C}-9\right)$ & & $37.31\left(\mathrm{CH}_{2}, \mathrm{C}-9\right)$ & \\
\hline $37.31\left(\mathrm{CH}_{2}, \mathrm{C}-17\right)$ & $38.28\left(\mathrm{CH}_{2}, \mathrm{C}-9^{\prime}\right)$ & $30.25\left(\mathrm{CH}_{2}, \mathrm{C}-17\right)$ & \\
\hline $27.32\left(\mathrm{CH}_{2}, \mathrm{C}-18\right)$ & & $28.19\left(\mathrm{CH}_{2}, \mathrm{C}-18\right)$ & \\
\hline $14.01\left(\mathrm{CH}_{2}, \mathrm{C}-19\right)$ & & $14.01\left(\mathrm{CH}_{2}, \mathrm{C}-19\right)$ & \\
\hline $34.37(\mathrm{C}, \mathrm{C}-4 \mathrm{a})$ & 34.22 (C,C-4a') & $34.33(\mathrm{C}, \mathrm{C}-4 \mathrm{a})$ & \\
\hline $34.15(\mathrm{C}, \mathrm{C}-12 \mathrm{a})$ & 34.18 (C,C-12a') & $34.18(\mathrm{C}, \mathrm{C}-12 \mathrm{a})$ & \\
\hline $31.82\left(\mathrm{CH}_{3}, \mathrm{C}-12 \mathrm{~b}\right)$ & $31.91\left(\mathrm{CH}_{3}, \mathrm{C}-4 \mathrm{~b}^{\prime}\right)$ & $31.87\left(\mathrm{CH}_{3}, \mathrm{C}-4 \mathrm{~b}\right)$ & \\
\hline $31.62\left(\mathrm{CH}_{3}, \mathrm{C}-4 \mathrm{~b}\right)$ & $31.87\left(\mathrm{CH}_{3}, \mathrm{C}-12 \mathrm{~b}^{\prime}\right)$ & $31.81\left(\mathrm{CH}_{3}, \mathrm{C}-12 \mathrm{~b}\right)$ & \\
\hline
\end{tabular}


Table S2a. $\quad{ }^{1} \mathrm{H}-\mathrm{NMR}$-spectrum of $\mathbf{3}$ in $\mathrm{CDCl}_{3}$ at $269 \mathrm{~K}$.

\begin{tabular}{|c|c|c|}
\hline $\operatorname{paco}\left(\boldsymbol{\Delta}, \mathbf{\Delta}^{\prime}\right)$ & 1,2-alternate(0) & $\operatorname{cone}(\mathrm{M})$ \\
\hline 7.23 (s, br, 1H, H-11') & $7.28\left(\mathrm{~d},{ }^{4} \mathrm{~J}=2.2 \mathrm{~Hz}, 2 \mathrm{H}, \mathrm{H}-3\right)$ & $7.23(\mathrm{~s}, \mathrm{br}, \mathrm{H}-11)$ \\
\hline 7.22 (s, br, 1H, H-13') & $7.09\left(\mathrm{~d},{ }^{4} \mathrm{~J}=2.1 \mathrm{~Hz}, 2 \mathrm{H}, \mathrm{H}-5\right)$ & $6.80(\mathrm{~s}, \mathrm{br}, \mathrm{H}-13)$ \\
\hline $7.00(\mathrm{~s}, \mathrm{br}, 2 \mathrm{H}, \mathrm{H}-5,13)$ & $7.06\left(\mathrm{~d},{ }^{4} \mathrm{~J}=2.2 \mathrm{~Hz}, 2 \mathrm{H}, \mathrm{H}-11\right)$ & $6.80(\mathrm{~s}, \mathrm{br}, \mathrm{H}-3)$ \\
\hline 6.99 (s, br, 1H, H-3') & $6.87\left(\mathrm{~d},{ }^{4} \mathrm{~J}=2.2 \mathrm{~Hz}, 2 \mathrm{H}, \mathrm{H}-13\right)$ & 6.77 (s, br, H-5) \\
\hline \multicolumn{3}{|l|}{$6.96(\mathrm{~s}, \mathrm{br}, 1 \mathrm{H}, \mathrm{H}-11)$} \\
\hline \multicolumn{3}{|l|}{$6.84(\mathrm{~s}, \mathrm{br}, 1 \mathrm{H}, \mathrm{H}-3)$} \\
\hline \multicolumn{3}{|l|}{$6.74(\mathrm{~s}, \mathrm{br}, 1 \mathrm{H}, \mathrm{H}-5)$} \\
\hline $4.53\left(\mathrm{t},{ }^{3} \mathrm{~J}=7.8 \mathrm{~Hz}, \mathrm{H}-1\right)$ & $4.79\left(\mathrm{t},{ }^{3} \mathrm{~J}=9.0 \mathrm{~Hz}, 1 \mathrm{H}, \mathrm{H}-1\right)$ & $4.63(\mathrm{t}, \mathrm{br}, 1 \mathrm{H}, \mathrm{H}-1)$ \\
\hline $4.21\left(\mathrm{~d},{ }^{2} \mathrm{~J}=12.8 \mathrm{~Hz}, \mathrm{H}-9 \mathrm{a}\right)$ & $4.10\left(\mathrm{~d},{ }^{2} \mathrm{~J}=13.3 \mathrm{~Hz}, 1 \mathrm{H}, \mathrm{H}-17 \mathrm{a}\right)$ & $4.32\left(\mathrm{~d},{ }^{2} \mathrm{~J}=11.2 \mathrm{~Hz}, \mathrm{H}-9 \mathrm{a}\right)$ \\
\hline $3.91\left(\mathrm{~d},{ }^{2} \mathrm{~J}=15.4 \mathrm{~Hz}, \mathrm{H}-9 \mathrm{a}^{\prime}\right)$ & $3.16(\mathrm{~d}, 1 \mathrm{H}, \mathrm{H}-17 \mathrm{~b})$ & $3.19\left(\mathrm{~d},{ }^{2} \mathrm{~J} \sim 12 \mathrm{~Hz}, \mathrm{H}-9 \mathrm{~b}\right)$ \\
\hline $3.87\left(\mathrm{~d},{ }^{2} \mathrm{~J}=15.4 \mathrm{~Hz}, \mathrm{H}-17 \mathrm{a}\right)$ & $3.92\left(\mathrm{~d},{ }^{2} \mathrm{~J} \sim 16 \mathrm{~Hz}, 2 \mathrm{H}, \mathrm{H}-9 \mathrm{a}\right)$ & $4.13(\mathrm{H}-17 \mathrm{a})$ \\
\hline $3.82\left(\mathrm{~d}^{2} \mathrm{~J} \sim 15 \mathrm{~Hz}, \mathrm{H}-9 \mathrm{~b}^{\prime}\right)$ & $3.79\left(\mathrm{~d},{ }^{2} \mathrm{~J} \sim 16 \mathrm{~Hz}, 2 \mathrm{H}, \mathrm{H}-9 \mathrm{~b}\right)$ & $3.18(\mathrm{H}-17 \mathrm{~b})$ \\
\hline \multicolumn{3}{|l|}{$3.78\left(\mathrm{~d},{ }^{2} \mathrm{~J}=15.3 \mathrm{~Hz}, \mathrm{H}-17 \mathrm{~b}\right)$} \\
\hline \multicolumn{3}{|l|}{$3.19\left(\mathrm{~d},{ }^{2} \mathrm{~J}=13.0 \mathrm{~Hz}, \mathrm{H}-9 \mathrm{~b}\right)$} \\
\hline $3.57(\mathrm{~s}, 3 \mathrm{H}, \mathrm{H}-8)$ & $3.13(\mathrm{~s}, 6 \mathrm{H}, \mathrm{H}-8)$ & $3.88(\mathrm{H}-8)$ \\
\hline $3.57\left(\mathrm{~s}, 3 \mathrm{H}, \mathrm{H}-8^{\prime}\right)$ & $2.91(\mathrm{~s}, 6 \mathrm{H}, \mathrm{H}-16)$ & $3.87(\mathrm{H}-16)$ \\
\hline \multicolumn{3}{|l|}{$3.43(\mathrm{~s}, 3 \mathrm{H}, \mathrm{H}-16)$} \\
\hline \multicolumn{3}{|l|}{$2.02\left(\mathrm{~s}, 3 \mathrm{H}, \mathrm{H}-16^{\prime}\right)$} \\
\hline $1.38(\mathrm{~s}, 9 \mathrm{H}, \mathrm{H}-12 \mathrm{~b} ')$ & $1.29(\mathrm{~s}, 18 \mathrm{H}, \mathrm{H}-4 \mathrm{~b})$ & $1.22(\mathrm{H}-4 \mathrm{~b})$ \\
\hline 1.23 (s, 9H, H-4b') & $1.25(\mathrm{~s}, 18 \mathrm{H}, \mathrm{H}-12 \mathrm{~b})$ & $1.09(\mathrm{H}-12 \mathrm{~b})$ \\
\hline \multicolumn{3}{|l|}{$1.22(\mathrm{~s}, 9 \mathrm{H}, \mathrm{H}-12 \mathrm{~b})$} \\
\hline \multicolumn{3}{|l|}{1.09 (s, 9H, H-4b) } \\
\hline $2.00(\mathrm{~m}, 2 \mathrm{H}, \mathrm{H}-18)$ & $2.05(\mathrm{~m}, 2 \mathrm{H}, \mathrm{H}-18)$ & $2.00(\mathrm{H}-18)$ \\
\hline $1.46(\mathrm{~m}, 2 \mathrm{H}, \mathrm{H}-19)$ & $1.39(\mathrm{H}-19)$ & $1.46(\mathrm{H}-19)$ \\
\hline $1.01\left(\mathrm{t}, 3 \mathrm{H},{ }^{3} \mathrm{~J}=7.2 \mathrm{~Hz}, \mathrm{H}-20\right)$ & $0.96\left(\mathrm{t}, 3 \mathrm{H},{ }^{3} \mathrm{~J}=7.2 \mathrm{~Hz}, \mathrm{H}-20\right)$ & $1.01\left(\mathrm{t},{ }^{3} \mathrm{~J}=7.2 \mathrm{~Hz}, \mathrm{H}-20\right)$ \\
\hline
\end{tabular}


Table S2b. $\quad{ }^{13} \mathrm{C}-\mathrm{NMR}$-spectrum of $\mathbf{3}$ in $\mathrm{CDCl}_{3}$ at $269 \mathrm{~K}$.

\begin{tabular}{|c|c|c|c|}
\hline \multicolumn{2}{|c|}{$\operatorname{paco}\left(\mathbf{\Lambda}^{\prime}, \mathbf{\Delta}^{\prime}\right)$} & \multirow{2}{*}{$\begin{array}{r}\text { 1,2-alternate(0) } \\
15364(C-7)\end{array}$} & \multirow{3}{*}{$\begin{array}{r}\text { cone(M) } \\
152.2\left(\mathrm{br}, \mathrm{C}_{\mathrm{i}}-7\right)\end{array}$} \\
\hline $154.56\left(\mathrm{C}_{\mathrm{i}}-7\right)$ & $154.98\left(\mathrm{C}_{\mathrm{i}}-7^{\prime}\right)$ & & \\
\hline $154.82\left(\mathrm{C}_{\mathrm{i}}-15\right)$ & $154.56\left(\mathrm{C}_{\mathrm{i}}-15^{\prime}\right)$ & $154.61\left(\mathrm{C}_{\mathrm{i}}-15\right)$ & \\
\hline $144.49\left(\mathrm{C}_{\mathrm{i}}-4\right)$ & $144.91\left(\mathrm{C}_{\mathrm{i}}-4^{\prime}\right)$ & $143.96\left(\mathrm{C}_{\mathrm{i}}-4\right)$ & $144.35\left(\mathrm{C}_{\mathrm{i}}-4\right)$ \\
\hline $144.91\left(\mathrm{C}_{\mathrm{i}}-12\right)$ & $143.22\left(\mathrm{C}_{\mathrm{i}}-12^{\prime}\right)$ & $144.43\left(\mathrm{C}_{\mathrm{i}}-12\right)$ & $144.35\left(\mathrm{C}_{\mathrm{i}}-12\right)$ \\
\hline $136.91\left(C_{i}-2\right)$ & $138.05\left(\mathrm{C}_{\mathrm{i}-2} \mathrm{2}^{\prime}\right)$ & $138.09\left(C_{i}-2\right)$ & $137.22\left(\mathrm{C}_{\mathrm{i}}-2\right)$ \\
\hline $135.30\left(\mathrm{C}_{\mathrm{i}}-10\right)$ & $133.11\left(\mathrm{C}_{\mathrm{i}}-10^{\prime}\right)$ & $135.10\left(\mathrm{C}_{\mathrm{i}}-14\right)$ & $133.84\left(\mathrm{C}_{\mathrm{i}}-14\right)$ \\
\hline $133.27\left(\mathrm{C}_{\mathrm{i}}-14\right)$ & $133.11\left(\mathrm{C}_{\mathrm{i}}-14^{\prime}\right)$ & $132.68\left(\mathrm{C}_{\mathrm{i}}-10\right)$ & $133.83\left(\mathrm{C}_{\mathrm{i}}-10\right)$ \\
\hline $132.78\left(\mathrm{C}_{\mathrm{i}}-6\right)$ & $133.72\left(\mathrm{C}_{\mathrm{i}}-6^{\prime}\right)$ & $132.60\left(\mathrm{C}_{\mathrm{i}}-6\right)$ & $133.52\left(\mathrm{C}_{\mathrm{i}}-6\right)$ \\
\hline $125.61(\mathrm{CH}, \mathrm{C}-13)$ & $126.36\left(\mathrm{CH}, \mathrm{C}-13^{\prime}\right)$ & $126.11(\mathrm{CH}, \mathrm{C}-5)$ & $126.26(\mathrm{CH}, \mathrm{C}-5)$ \\
\hline $125.10(\mathrm{CH}, \mathrm{C}-11)$ & 126.27 (CH, C-11') & $125.87(\mathrm{CH}, \mathrm{C}-11)$ & $124.48(\mathrm{CH}, \mathrm{C}-11)$ \\
\hline $124.49(\mathrm{CH}, \mathrm{C}-5)$ & $125.37\left(\mathrm{CH}, \mathrm{C}-5^{\prime}\right)$ & $125.30(\mathrm{CH}, \mathrm{C}-13)$ & $124.02(\mathrm{CH}, \mathrm{C}-13)$ \\
\hline $121.93(\mathrm{CH}, \mathrm{C}-3)$ & $121.93\left(\mathrm{CH}, \mathrm{C}-3^{\prime}\right)$ & $122.13(\mathrm{CH}, \mathrm{C}-3)$ & $121.52(\mathrm{CH}, \mathrm{C}-3)$ \\
\hline $61.22\left(\mathrm{CH}_{3}, \mathrm{C}-8\right)$ & $59.89\left(\mathrm{CH}_{3}, \mathrm{C}-8^{\prime}\right)$ & $60.63\left(\mathrm{CH}_{3}, \mathrm{C}-8\right)$ & $61.23\left(\mathrm{CH}_{3}, \mathrm{C}-8\right)$ \\
\hline $60.23\left(\mathrm{CH}_{3}, \mathrm{C}-16\right)$ & $58.36\left(\mathrm{CH}_{3}, \mathrm{C}-16^{\prime}\right)$ & $59.89\left(\mathrm{CH}_{3}, \mathrm{C}-16\right)$ & $61.21\left(\mathrm{CH}_{3}, \mathrm{C}-16\right)$ \\
\hline $35.10(\mathrm{CH}, \mathrm{C}-1)$ & & $34.31(\mathrm{CH}, \mathrm{C}-1)$ & $35.56(\mathrm{CH}, \mathrm{C}-1)$ \\
\hline $30.98\left(\mathrm{CH}_{2}, \mathrm{C}-9\right)$ & $37.84\left(\mathrm{CH}_{2}, \mathrm{C}-9^{\prime}\right)$ & $36.88\left(\mathrm{CH}_{2}, \mathrm{C}-9\right)$ & $36.98\left(\mathrm{CH}_{2}, \mathrm{C}-9\right)$ \\
\hline $36.83\left(\mathrm{CH}_{2}, \mathrm{C}-17\right)$ & & $30.06\left(\mathrm{CH}_{2}, \mathrm{C}-17\right)$ & $30.98\left(\mathrm{CH}_{2}, \mathrm{C}-17\right)$ \\
\hline $36.15\left(\mathrm{CH}_{2}, \mathrm{C}-18\right)$ & & $37.09\left(\mathrm{CH}_{2}, \mathrm{C}-18\right)$ & $36.15\left(\mathrm{CH}_{2}, \mathrm{C}-18\right)$ \\
\hline $21.53\left(\mathrm{CH}_{2}, \mathrm{C}-19\right)$ & & $21.71\left(\mathrm{CH}_{2}, \mathrm{C}-19\right)$ & $21.53\left(\mathrm{CH}_{2}, \mathrm{C}-19\right)$ \\
\hline $14.39\left(\mathrm{CH}_{3}, \mathrm{C}-20\right)$ & & $14.23\left(\mathrm{CH}_{3}, \mathrm{C}-20\right)$ & $14.39\left(\mathrm{CH}_{2}, \mathrm{C}-20\right)$ \\
\hline 33.89 (C,C-4a) & 33.89 (C,C-4a') & $34.02(\mathrm{C}, \mathrm{C}-4 \mathrm{a})$ & not discriminable \\
\hline $33.89(\mathrm{C}, \mathrm{C}-12 \mathrm{a})$ & 33.89 (C,C-12a') & $33.89(\mathrm{C}, \mathrm{C}-12 \mathrm{a})$ & \\
\hline $31.27\left(\mathrm{CH}_{3}, \mathrm{C}-4 \mathrm{~b}\right)$ & $31.41\left(\mathrm{CH}_{3}, \mathrm{C}-4 \mathrm{~b}^{\prime}\right)$ & $31.48\left(\mathrm{CH}_{3}, \mathrm{C}-4 \mathrm{~b}\right)$ & \\
\hline $31.36\left(\mathrm{CH}_{3}, \mathrm{C}-12 \mathrm{~b}\right)$ & $31.52\left(\mathrm{CH}_{3}, \mathrm{C}-12 \mathrm{~b}^{\prime}\right)$ & $31.36\left(\mathrm{CH}_{3}, \mathrm{C}-12 \mathrm{~b}\right)$ & \\
\hline
\end{tabular}


Table S2c. $\quad{ }^{1} \mathrm{H}-\mathrm{NMR}-$ spectrum of $\mathbf{3}$ in $\mathrm{TCl}-\mathrm{d}_{2}$ at $265 \mathrm{~K}$.

\begin{tabular}{|c|c|c|}
\hline $\operatorname{paco}\left(\boldsymbol{\Delta}, \mathbf{\Delta}^{\prime}\right)$ & 1,2-alternate(0) & cone $(\mathrm{M})$ \\
\hline $7.08\left(\mathrm{~s}, \mathrm{br}, 1 \mathrm{H}, \mathrm{H}-11^{\prime}\right)$ & $7.08(\mathrm{H}-3)$ & $7.11(\mathrm{H}-11)$ \\
\hline 7.06 (s, br, 1H, H-13') & $6.93\left(\mathrm{~d},{ }^{4} \mathrm{~J}=2.2 \mathrm{~Hz}, 2 \mathrm{H}, \mathrm{H}-5\right)$ & $6.69(\mathrm{H}-13)$ \\
\hline $6.94\left(\mathrm{~s}, \mathrm{br}, 1 \mathrm{H}, \mathrm{H}-3^{\prime}\right)$ & $6.92\left(\mathrm{~d},{ }^{4} \mathrm{~J}=2.0 \mathrm{~Hz}, 2 \mathrm{H}, \mathrm{H}-11\right)$ & $6.66(\mathrm{H}-3)$ \\
\hline $6.88(\mathrm{~s}, \mathrm{br}, 1 \mathrm{H}, \mathrm{H}-11)$ & $6.77(\mathrm{~d}, \mathrm{~J}=1.9 \mathrm{~Hz}, 2 \mathrm{H}, \mathrm{H}-13)$ & $6.61(\mathrm{H}-5)$ \\
\hline \multicolumn{3}{|l|}{$6.85\left(\mathrm{~s}, \mathrm{br}, 2 \mathrm{H}, \mathrm{H}-13,5^{\prime}\right)$} \\
\hline \multicolumn{3}{|l|}{$6.61(\mathrm{~s}, \mathrm{br}, 1 \mathrm{H}, \mathrm{H}-3)$} \\
\hline \multicolumn{3}{|l|}{$6.53(\mathrm{~s}, \mathrm{br}, 1 \mathrm{H}, \mathrm{H}-5)$} \\
\hline $4.40\left(\mathrm{t},{ }^{3} \mathrm{~J}=7.7 \mathrm{~Hz}, \mathrm{H}-1\right)$ & $4.55\left(\mathrm{t},{ }^{3} \mathrm{~J}=8.0 \mathrm{~Hz}, 1 \mathrm{H}, \mathrm{H}-1\right)$ & $4.46(\mathrm{H}-1)$ \\
\hline $4.07\left(\mathrm{~d},{ }^{2} \mathrm{~J}=12.6 \mathrm{~Hz}, \mathrm{H}-9 \mathrm{a}\right)$ & $3.97\left(\mathrm{~d},{ }^{2} \mathrm{~J}=13.0 \mathrm{~Hz}, 1 \mathrm{H}, \mathrm{H}-17 \mathrm{a}\right)$ & $4.13(\mathrm{H}-9 \mathrm{a})$ \\
\hline $3.74\left(\mathrm{~d},{ }^{2} \mathrm{~J}=15.7 \mathrm{~Hz}, \mathrm{H}-17 \mathrm{a}\right)$ & $3.74\left(\mathrm{~d},{ }^{2} \mathrm{~J} \sim 15.5 \mathrm{~Hz}, 2 \mathrm{H}, \mathrm{H}-9 \mathrm{a}\right)$ & $3.03(\mathrm{H}-9 \mathrm{~b})$ \\
\hline $3.68\left(\mathrm{~d},{ }^{2} \mathrm{~J} \sim 15 \mathrm{~Hz}, \mathrm{H}-17 \mathrm{~b}\right)$ & $3.65\left(\mathrm{~d},{ }^{2} \mathrm{~J}=15.9 \mathrm{~Hz}, 2 \mathrm{H}, \mathrm{H}-9 \mathrm{~b}\right)$ & $4.00(\mathrm{H}-17 \mathrm{a})$ \\
\hline $3.07\left(\mathrm{~d},{ }^{2} \mathrm{~J}=12.8 \mathrm{~Hz}, \mathrm{H}-9 \mathrm{~b}\right)$ & $3.03\left(\mathrm{~d},{ }^{2} \mathrm{~J}=13.7 \mathrm{~Hz}, 1 \mathrm{H}, \mathrm{H}-17 \mathrm{~b}\right)$ & $3.06(\mathrm{H}-17 \mathrm{~b})$ \\
\hline \multicolumn{3}{|l|}{$3.73\left(\mathrm{~d}^{2}{ }^{2} \mathrm{~J} \sim 10 \mathrm{~Hz}, \mathrm{H}-9 \mathrm{a}{ }^{\prime}\right)$} \\
\hline \multicolumn{3}{|l|}{$3.70\left(\mathrm{~d}^{2}{ }^{2} \mathrm{~J} \sim 10 \mathrm{~Hz}, \mathrm{H}-9 \mathrm{~b}^{\prime}\right)$} \\
\hline $3.49(\mathrm{~s}, 3 \mathrm{H}, \mathrm{H}-8)$ & $2.97(\mathrm{~s}, 6 \mathrm{H}, \mathrm{H}-8)$ & $3.77(\mathrm{H}-8)$ \\
\hline $3.40\left(\mathrm{~s}, 3 \mathrm{H}, \mathrm{H}-8^{\prime}\right)$ & $2.78(\mathrm{~s}, 6 \mathrm{H}, \mathrm{H}-16)$ & $3.75(\mathrm{H}-16)$ \\
\hline \multicolumn{3}{|l|}{$3.27(\mathrm{~s}, 3 \mathrm{H}, \mathrm{H}-16)$} \\
\hline \multicolumn{3}{|l|}{$1.62\left(\mathrm{~s}, 3 \mathrm{H}, \mathrm{H}-16^{\prime}\right)$} \\
\hline 1.27 (s, 9H, H-12b') & $1.16(\mathrm{~s}, 18 \mathrm{H}, \mathrm{H}-4 \mathrm{~b})$ & $1.27(\mathrm{H}-12 \mathrm{~b})$ \\
\hline $1.13\left(\mathrm{~s}, 18 \mathrm{H}, \mathrm{H}-4 \mathrm{~b}^{\prime}, 12 \mathrm{~b}^{\prime}\right)$ & $1.13(\mathrm{~s}, \mathrm{H}-12 \mathrm{~b})$ & $1.13(\mathrm{H}-4 \mathrm{~b})$ \\
\hline \multicolumn{3}{|l|}{$0.92(\mathrm{~s}, 9 \mathrm{H}, \mathrm{H}-4 \mathrm{~b})$} \\
\hline $1.94(\mathrm{~m}, 1 \mathrm{H}, \mathrm{H}-18 \mathrm{a})$ & $1.89(\mathrm{~m}, 1 \mathrm{H}, \mathrm{H}-18)$ & $1.94(\mathrm{H}-18 \mathrm{a})$ \\
\hline $1.83(\mathrm{~m}, 1 \mathrm{H}, \mathrm{H}-18 \mathrm{~b})$ & $1.27(\mathrm{~m}, 1 \mathrm{H}, \mathrm{H}-19)$ & $1.83(\mathrm{H}-18 \mathrm{~b})$ \\
\hline $1.35(\mathrm{~m}, 2 \mathrm{H}, \mathrm{H}-19)$ & $0.85\left(\mathrm{t}, 3 \mathrm{H},{ }^{3} \mathrm{~J}=7.3 \mathrm{~Hz}, \mathrm{H}-20\right)$ & $1.35(\mathrm{H}-19)$ \\
\hline $0.90\left(\mathrm{t}, 3 \mathrm{H},{ }^{3} \mathrm{~J}=7.3 \mathrm{~Hz}, \mathrm{H}-20\right)$ & & $0.90(\mathrm{H}-20)$ \\
\hline
\end{tabular}


Table S2d. $\quad{ }^{13} \mathrm{C}-\mathrm{NMR}-$ spectrum of $\mathbf{3}$ in $\mathrm{TCl}-\mathrm{d}_{2}$ at $265 \mathrm{~K}$.

\begin{tabular}{|c|c|c|c|}
\hline \multicolumn{2}{|c|}{$\operatorname{paco}\left(\boldsymbol{\Delta}, \mathbf{\Delta}^{\prime}\right)$} & \multirow{2}{*}{$\begin{array}{c}\text { 1,2-alternate }(\mathbf{O}) \\
153.94\left(\mathrm{C}_{\mathrm{i}}-7\right)\end{array}$} & \multirow[t]{3}{*}{ cone $(\mathrm{M})$} \\
\hline $154.74\left(\mathrm{C}_{\mathrm{i}}-7\right)$ & $155.34\left(\mathrm{C}_{\mathrm{i}} \mathbf{7}^{\prime}\right)$ & & \\
\hline $155.07\left(\mathrm{C}_{\mathrm{i}}-15\right)$ & $154.73\left(\mathrm{C}_{\mathrm{i}}-15^{\prime}\right)$ & $154.69\left(\mathrm{C}_{\mathrm{i}}-15\right)$ & \\
\hline $144.55\left(\mathrm{C}_{\mathrm{i}}-4\right)$ & $145.34\left(\mathrm{C}_{\mathrm{i}}-4^{\prime}\right)$ & $144.22\left(\mathrm{C}_{\mathrm{i}}-4\right)$ & \\
\hline $145.27\left(\mathrm{C}_{\mathrm{i}}-12\right)$ & $143.54\left(\mathrm{C}_{\mathrm{i}}-12^{\prime}\right)$ & $144.55\left(\mathrm{C}_{\mathrm{i}}-12\right)$ & \\
\hline $137.07\left(\mathrm{C}_{\mathrm{i}}-2\right)$ & $138.61\left(\mathrm{C}_{\mathrm{i}}-2^{\prime}\right)$ & $138.45\left(\mathrm{C}_{\mathrm{i}}-2\right)$ & \\
\hline $136.15\left(\mathrm{C}_{\mathrm{i}}-10\right)$ & $133.36\left(\mathrm{C}_{\mathrm{i}}-10^{\prime}\right)$ & $132.95\left(\mathrm{C}_{\mathrm{i}}-10\right)$ & \\
\hline $133.12\left(\mathrm{C}_{\mathrm{i}}-14\right)$ & $133.36\left(\mathrm{C}_{\mathrm{i}}-14^{\prime}\right)$ & $135.32\left(\mathrm{C}_{\mathrm{i}}-14\right)$ & \\
\hline $133.01\left(\mathrm{C}_{\mathrm{i}}-6\right)$ & $133.01\left(\mathrm{C}_{\mathrm{i}}-6^{\prime}\right)$ & $132.81\left(\mathrm{C}_{\mathrm{i}}-6\right)$ & \\
\hline $125.91(\mathrm{CH}, \mathrm{C}-13)$ & $126.63\left(\mathrm{CH}, \mathrm{C}-11^{\prime}\right)$ & $126.39(\mathrm{CH}, \mathrm{C}-5)$ & $126.6(\mathrm{CH}, \mathrm{C}-5)$ \\
\hline $125.62(\mathrm{CH}, \mathrm{C}-11)$ & $(\mathrm{CH}, \mathrm{C}-13$ ') & $126.30(\mathrm{CH}, \mathrm{C}-11)$ & $124.8(\mathrm{CH}, \mathrm{C}-11)$ \\
\hline $124.83(\mathrm{CH}, \mathrm{C}-5)$ & 125.57 (CH,C-5') & $125.74(\mathrm{CH}, \mathrm{C}-13)$ & $124.3(\mathrm{CH}, \mathrm{C}-13)$ \\
\hline $122.60(\mathrm{CH}, \mathrm{C}-3)$ & $122.08\left(\mathrm{CH}, \mathrm{C}-3^{\prime}\right)$ & $122.60(\mathrm{CH}, \mathrm{C}-3)$ & $122.0(\mathrm{CH}, \mathrm{C}-3)$ \\
\hline $61.87\left(\mathrm{CH}_{3}, \mathrm{C}-8\right)$ & $60.28\left(\mathrm{CH}_{3}, \mathrm{C}-8^{\prime}\right)$ & $60.82\left(\mathrm{CH}_{3}, \mathrm{C}-8\right)$ & $61.6\left(b r, \mathrm{CH}_{3}\right.$ \\
\hline $60.52\left(\mathrm{CH}_{3}, \mathrm{C}-16\right)$ & $58.77\left(\mathrm{CH}_{3}, \mathrm{C}-16^{\prime}\right)$ & $60.33\left(\mathrm{CH}_{3}, \mathrm{C}-16\right)$ & C-8;16) \\
\hline $35.46(\mathrm{CH}, \mathrm{C}-1)$ & & $34.60(\mathrm{CH}, \mathrm{C}-1)$ & $36.0(b r, \mathrm{CH}, \mathrm{C}-1)$ \\
\hline $31.36\left(\mathrm{CH}_{2}, \mathrm{C}-9\right)$ & & $37.35\left(\mathrm{CH}_{2}, \mathrm{C}-9\right)$ & \\
\hline $37.26\left(\mathrm{CH}_{2}, \mathrm{C}-17\right)$ & $38.25\left(\mathrm{CH}_{2}, \mathrm{C}-9^{\prime}\right)$ & $30.48\left(\mathrm{CH}_{2}, \mathrm{C}-17\right)$ & \\
\hline $36.57\left(\mathrm{CH}_{2}, \mathrm{C}-18\right)$ & & $37.35\left(\mathrm{CH}_{2}, \mathrm{C}-18\right)$ & \\
\hline $22.01\left(\mathrm{CH}_{2}, \mathrm{C}-19\right)$ & & $22.28\left(\mathrm{CH}_{2}, \mathrm{C}-19\right)$ & \\
\hline $14.90\left(\mathrm{CH}_{3}, \mathrm{C}-20\right)$ & & $14.77\left(\mathrm{CH}_{3}, \mathrm{C}-20\right)$ & \\
\hline $34.13(\mathrm{C}, \mathrm{C}-4 \mathrm{a})$ & 34.23 (C,C-4a') & $34.32(\mathrm{C}, \mathrm{C}-4 \mathrm{a})$ & \\
\hline 34.38 (C,C-12a) & 34.19 (C,C-12a') & $34.19(\mathrm{C}, \mathrm{C}-12 \mathrm{a})$ & \\
\hline $31.60\left(\mathrm{CH}_{3}, \mathrm{C}-4 \mathrm{~b}\right)$ & $31.88\left(\mathrm{CH}_{3}, \mathrm{C}-4 \mathrm{~b}^{\prime}\right)$ & $31.82\left(\mathrm{CH}_{3}, \mathrm{C}-4 \mathrm{~b}\right)$ & \\
\hline $31.82\left(\mathrm{CH}_{3}, \mathrm{C}-12 \mathrm{~b}\right)$ & $31.88\left(\mathrm{CH}_{3}, \mathrm{C}-12 \mathrm{~b}^{\prime}\right)$ & $31.88\left(\mathrm{CH}_{3}, \mathrm{C}-12 \mathrm{~b}\right)$ & \\
\hline
\end{tabular}


Table S3a. ${ }^{1} \mathrm{H}-\mathrm{NMR}$-spectrum of 4 in $\mathrm{CDCl}_{3}$ at $265 \mathrm{~K}$.

\begin{tabular}{|c|c|c|}
\hline $\operatorname{paco}\left(\boldsymbol{\Delta}, \boldsymbol{\Delta}^{\prime}\right)$ & 1,2-alternate(0) & cone $(\mathrm{M})$ \\
\hline $7.21\left((\mathrm{~d}), 1 \mathrm{H}, \mathrm{H}-11^{\prime}\right)$ & $7.26(\mathrm{~d}, \mathrm{H}-3)$ & $7.20(\mathrm{H}-11)$ \\
\hline $7.20\left((\mathrm{~d}), 1 \mathrm{H}, \mathrm{H}-11^{\prime}\right)$ & $7.07\left(\mathrm{~d},{ }^{4} \mathrm{~J}=2.4 \mathrm{~Hz}, 2 \mathrm{H}, \mathrm{H}-5\right)$ & $6.78(\mathrm{H}-13)$ \\
\hline $6.98\left(((\mathrm{~d}), 1 \mathrm{H}, \mathrm{H}-5)^{\prime}\right)$ & $7.04(\mathrm{~d}, \mathrm{~J}=2.3 \mathrm{~Hz}, 2 \mathrm{H}, \mathrm{H}-11)$ & $6.78(\mathrm{H}-3)$ \\
\hline $6.97\left((\mathrm{~d}), 2 \mathrm{H}, \mathrm{H}-3^{\prime}\right)$ & $6.86(\mathrm{~d}, \mathrm{~J}=2.4 \mathrm{~Hz}, 2 \mathrm{H}, \mathrm{H}-13)$ & $6.75(\mathrm{H}-5)$ \\
\hline \multicolumn{3}{|l|}{$6.97((\mathrm{~d}), 1 \mathrm{H}, \mathrm{H}-13)$} \\
\hline \multicolumn{3}{|l|}{$6.94(\mathrm{~d}), 1 \mathrm{H}, \mathrm{H}-11)$} \\
\hline \multicolumn{3}{|l|}{$6.81((\mathrm{~d}), 1 \mathrm{H}, \mathrm{H}-3)$} \\
\hline \multicolumn{3}{|l|}{$6.72((\mathrm{~d}), 1 \mathrm{H}, \mathrm{H}-5)$} \\
\hline $4.47\left(\mathrm{t},{ }^{3} \mathrm{~J}=7.9 \mathrm{~Hz}, \mathrm{H}-1\right)$ & $4.73\left(\mathrm{t},{ }^{3} \mathrm{~J}=8.2 \mathrm{~Hz}, 1 \mathrm{H}, \mathrm{H}-1\right)$ & $4.57((\mathrm{t}), \mathrm{H}-1)$ \\
\hline $4.18\left(\mathrm{~d},{ }^{2} \mathrm{~J}=12.8 \mathrm{~Hz}, \mathrm{H}-9 \mathrm{a}\right)$ & $4.08\left(\mathrm{~d},{ }^{2} \mathrm{~J}=13.3 \mathrm{~Hz}, 1 \mathrm{H}, \mathrm{H}-17 \mathrm{a}\right)$ & 4.30 (br, H-9a) \\
\hline $3.86\left(\mathrm{~d},{ }^{2} \mathrm{~J}=14.0 \mathrm{~Hz}, \mathrm{H}-17 \mathrm{a}\right)$ & $3.87\left(\mathrm{~d},{ }^{2} \mathrm{~J}=14.7 \mathrm{~Hz}, 2 \mathrm{H}, \mathrm{H}-9 \mathrm{a}\right)$ & 3.17 (br, H-9b) \\
\hline $3.84\left(\mathrm{~d},{ }^{2} \mathrm{~J}=13.0 \mathrm{~Hz}, \mathrm{H}-9 \mathrm{a}\right)$ & $3.75(\mathrm{~d}, 2 \mathrm{H}, \mathrm{H}-9 \mathrm{~b})$ & not discriminable \\
\hline \multicolumn{3}{|l|}{$3.83\left(\mathrm{~d},{ }^{2} \mathrm{~J}=14.2, \mathrm{H}-17 \mathrm{~b}\right)$} \\
\hline \multicolumn{3}{|l|}{$3.80\left(\mathrm{~d},{ }^{2} \mathrm{~J}=13.8 \mathrm{~Hz}, \mathrm{H}-9 \mathrm{~b}^{\prime}\right)$} \\
\hline \multicolumn{3}{|l|}{$3.17\left(\mathrm{~d},{ }^{2} \mathrm{~J}=13.0 \mathrm{~Hz}, \mathrm{H}-9 \mathrm{~b}\right)$} \\
\hline $3.55(\mathrm{~s}, 3 \mathrm{H}, \mathrm{H}-8)$ & $3.11(\mathrm{~s}, 6 \mathrm{H}, \mathrm{H}-8)$ & $3.88(\mathrm{H}-8)$ \\
\hline $3.55\left(\mathrm{~s}, 3 \mathrm{H}, \mathrm{H}-8^{\prime}\right)$ & $2.89(\mathrm{~s}, 6 \mathrm{H}, \mathrm{H}-16)$ & $3.83(\mathrm{H}-16)$ \\
\hline \multicolumn{3}{|l|}{$3.40(\mathrm{~s}, 3 \mathrm{H}, \mathrm{H}-16)$} \\
\hline \multicolumn{3}{|l|}{$2.00\left(\mathrm{~s}, 3 \mathrm{H}, \mathrm{H}-16^{\prime}\right)$} \\
\hline $1.36\left(\mathrm{~s}, 9 \mathrm{H}, \mathrm{H}-12 \mathrm{~b}^{\prime}\right)$ & $1.27(\mathrm{~s}, 18 \mathrm{H}, \mathrm{H}-4 \mathrm{~b})$ & not discriminable \\
\hline $1.20(\mathrm{~s}, 9 \mathrm{H}, \mathrm{H}-4 \mathrm{~b})$ & $1.23(\mathrm{~s}, 18 \mathrm{H}, \mathrm{H}-12 \mathrm{~b})$ & \\
\hline \multicolumn{3}{|l|}{$1.20(\mathrm{~s}, 9 \mathrm{H}, \mathrm{H}-12 \mathrm{~b})$} \\
\hline \multicolumn{3}{|l|}{1.07 (s, 9H, H-4b) } \\
\hline $1.97(\mathrm{~m}, \mathrm{H}-18)$ & $2.03(\mathrm{~m}, \mathrm{H}-18)$ & $1.97(\mathrm{~m}, \mathrm{H}-18)$ \\
\hline $1.41(\mathrm{~m}, \mathrm{H}-19)$ & $1.34(\mathrm{~m}, \mathrm{H}-19)$ & not discriminable \\
\hline $1.39(\mathrm{~m}, \mathrm{H}-20)$ & $1.39(\mathrm{~m}, \mathrm{H}-20)$ & \\
\hline $1.32(\mathrm{~m}, \mathrm{H}-21)$ & $1.31(\mathrm{~m}, \mathrm{H}-21)$ & \\
\hline $0.87\left(\mathrm{t},{ }^{3} \mathrm{~J}=7.0 \mathrm{~Hz}, 3 \mathrm{H}, \mathrm{H}-22\right)$ & $0.82(\mathrm{~m}, \mathrm{H}-22)$ & \\
\hline
\end{tabular}


Table S3b. ${ }^{13} \mathrm{C}-\mathrm{NMR}$-spectrum of $\mathbf{4}$ in $\mathrm{CDCl}_{3}$ at $265 \mathrm{~K}$.

\begin{tabular}{|c|c|c|c|}
\hline \multicolumn{2}{|c|}{$\operatorname{paco}\left(\boldsymbol{\Delta}, \boldsymbol{\Delta}^{\prime}\right)$} & \multirow{2}{*}{ 1,2-alternate(0) } & \multirow{3}{*}{$\begin{array}{c}\text { cone }(\mathrm{M}) \\
\text { not discriminable }\end{array}$} \\
\hline $154.83\left(\mathrm{C}_{\mathrm{i}}-15\right)$ & $154.99\left(\mathrm{C}_{\mathrm{i}^{-}} 7^{\prime}\right)$ & & \\
\hline $154.55\left(\mathrm{C}_{\mathrm{i}}-7\right)$ & $154.57\left(\mathrm{C}_{\mathrm{i}}-15^{\prime}\right)$ & $153.66\left(\mathrm{C}_{\mathrm{i}}-7\right)$ & \\
\hline $144.92\left(\mathrm{C}_{\mathrm{i}}-12\right)$ & $144.92\left(\mathrm{C}_{\mathrm{i}^{-}}-4^{\prime}\right)$ & $144.44\left(\mathrm{C}_{\mathrm{i}}-12\right)$ & not discriminable \\
\hline $144.50\left(C_{\mathrm{i}}-4\right)$ & $143.24\left(\mathrm{C}_{\mathrm{i}^{-}}-12^{\prime}\right)$ & $143.99\left(\mathrm{C}_{\mathrm{i}}-4\right)$ & \\
\hline $136.93\left(\mathrm{C}_{\mathrm{i}}-2\right)$ & $138.05\left(\mathrm{C}_{\mathrm{i}^{-}} 2^{\prime}\right)$ & $138.14\left(\mathrm{C}_{\mathrm{i}}-2\right)$ & not discriminable \\
\hline $135.32\left(\mathrm{C}_{\mathrm{i}}-10\right)$ & $133.12\left(\mathrm{C}_{\mathrm{i}^{-}}-10^{\prime}\right)$ & $135.09\left(\mathrm{C}_{\mathrm{i}}-14\right)$ & \\
\hline $133.28\left(\mathrm{C}_{\mathrm{i}}-6\right)$ & $133.12\left(\mathrm{C}_{\mathrm{i}^{-}}-14^{\prime}\right)$ & $132.69\left(\mathrm{C}_{\mathrm{i}}-10\right)$ & \\
\hline $132.78\left(\mathrm{C}_{\mathrm{i}}-14\right)$ & $132.75\left(\mathrm{C}_{\mathrm{i}}-6^{\prime}\right)$ & $132.61\left(\mathrm{C}_{\mathrm{i}}-6\right)$ & \\
\hline $125.61(\mathrm{CH}, \mathrm{C}-13)$ & $126.36\left(\mathrm{CH}, \mathrm{C}-13^{\prime}\right)$ & $126.11(\mathrm{CH}, \mathrm{C}-5)$ & 126.3 (br, C-11) \\
\hline $125.11(\mathrm{CH}, \mathrm{C}-11)$ & $126.27\left(\mathrm{CH}, \mathrm{C}-11^{\prime}\right)$ & $125.88(\mathrm{CH}, \mathrm{C}-11)$ & 124.5 (br, C-13) \\
\hline $124.50(\mathrm{CH}, \mathrm{C}-5)$ & $125.37\left(\mathrm{CH}, \mathrm{C}-5{ }^{\prime}\right)$ & $125.30(\mathrm{CH}, \mathrm{C}-13)$ & $124.1(\mathrm{br}, \mathrm{C}-5)$ \\
\hline $121.95(\mathrm{CH}, \mathrm{C}-3)$ & $121.91\left(\mathrm{CH}, \mathrm{C}-3^{\prime}\right)$ & $122.11(\mathrm{CH}, \mathrm{C}-3)$ & $121.7(\mathrm{br}, \mathrm{C}-3)$ \\
\hline $61.27\left(\mathrm{CH}_{3}, \mathrm{C}-8\right)$ & $59.91\left(\mathrm{CH}_{3}, \mathrm{C}-8^{\prime}\right)$ & $60.63\left(\mathrm{CH}_{3}, \mathrm{C}-8\right)$ & $61.3(\mathrm{C}-8)$ \\
\hline $60.24\left(\mathrm{CH}_{3}, \mathrm{C}-16\right)$ & $58.37\left(\mathrm{CH}_{3}, \mathrm{C}-16^{\prime}\right)$ & $59.91\left(\mathrm{CH}_{3}, \mathrm{C}-16\right)$ & $61.3(\mathrm{C}-16)$ \\
\hline $35.52(\mathrm{CH}, \mathrm{C}-1)$ & & $34.57(\mathrm{CH}, \mathrm{C}-1)$ & 36.1 (br, C-1) \\
\hline $36.82\left(\mathrm{CH}_{2}, \mathrm{C}-17\right)$ & $37.85\left(\mathrm{CH}_{2}, \mathrm{C}-9^{\prime}\right)$ & $36.90\left(\mathrm{CH}_{2}, \mathrm{C}-9\right)$ & 31.1 (br, C-9) \\
\hline $31.09\left(\mathrm{CH}_{2}, \mathrm{C}-9\right)$ & & $30.06\left(\mathrm{CH}_{2}, \mathrm{C}-17\right)$ & not discriminable \\
\hline $34.03\left(\mathrm{CH}_{2}, \mathrm{C}-18\right)$ & & $34.85\left(\mathrm{CH}_{2}, \mathrm{C}-18\right)$ & not discriminable \\
\hline $32.04\left(\mathrm{CH}_{2}, \mathrm{C}-19\right)$ & & $31.09\left(\mathrm{CH}_{2}, \mathrm{C}-19\right)$ & \\
\hline $28.21\left(\mathrm{CH}_{3}, \mathrm{C}-20\right)$ & & $28.30\left(\mathrm{CH}_{3}, \mathrm{C}-20\right)$ & \\
\hline $22.60\left(\mathrm{CH}_{3}, \mathrm{C}-21\right)$ & & $22.50\left(\mathrm{CH}_{3}, \mathrm{C}-21\right)$ & \\
\hline $14.32\left(\mathrm{CH}_{3}, \mathrm{C}-22\right)$ & & $14.22\left(\mathrm{CH}_{3}, \mathrm{C}-22\right)$ & \\
\hline $33.89(\mathrm{C}, \mathrm{C}-4 \mathrm{a})$ & 33.89 (C,C-4a') & $33.89(\mathrm{C}, \mathrm{C}-4 \mathrm{a})$ & not discriminable \\
\hline $33.89(\mathrm{C}, \mathrm{C}-12 \mathrm{a})$ & 33.89 (C,C-12a') & $33.89(\mathrm{C}, \mathrm{C}-12 \mathrm{a})$ & \\
\hline $31.42\left(\mathrm{CH}_{3}, \mathrm{C}-12 \mathrm{~b}\right)$ & $31.52\left(\mathrm{CH}_{3}, \mathrm{C}-12 \mathrm{~b}^{\prime}\right)$ & $31.48\left(\mathrm{CH}_{3}, \mathrm{C}-4 \mathrm{~b}\right)$ & \\
\hline $31.27\left(\mathrm{CH}_{3}, \mathrm{C}-4 \mathrm{~b}\right)$ & $31.42\left(\mathrm{CH}_{3}, \mathrm{C}-4 \mathrm{~b}^{\prime}\right)$ & $31.37\left(\mathrm{CH}_{3}, \mathrm{C}-12 \mathrm{~b}\right)$ & \\
\hline
\end{tabular}


Table S4a. $\quad{ }^{1} \mathrm{H}-\mathrm{NMR}$-spectrum of 5 in $\mathrm{CDCl}_{3}$ at $263 \mathrm{~K}$.

\begin{tabular}{|c|c|c|}
\hline $\operatorname{paco}\left(\boldsymbol{\Delta}, \mathbf{\Delta}^{\prime}\right)$ & 1,2-alternate(0) & cone $(\mathrm{M})$ \\
\hline $7.22\left((\mathrm{~d}), 1 \mathrm{H}, \mathrm{H}-11^{\prime}\right)$ & $7.26\left(\mathrm{~d},{ }^{4} \mathrm{~J}=2.4 \mathrm{~Hz}, 2 \mathrm{H} ; \mathrm{H}-3\right)$ & $7.21(\mathrm{H}-11)$ \\
\hline $7.21\left((\mathrm{~d}), 1 \mathrm{H}, \mathrm{H}-13^{\prime}\right)$ & $7.08\left(\mathrm{~d},{ }^{4} \mathrm{~J}=2.3 \mathrm{~Hz}, 2 \mathrm{H}, \mathrm{H}-5\right)$ & $6.79(\mathrm{H}-13)$ \\
\hline $6.99\left(1 \mathrm{H}, \mathrm{H}-5{ }^{\prime}\right)$ & $7.05\left(\mathrm{~d},{ }^{4} \mathrm{~J}=2.3 \mathrm{~Hz}, 2 \mathrm{H}, \mathrm{H}-11\right)$ & $6.79(\mathrm{H}-3)$ \\
\hline $6.98\left(1 \mathrm{H}, \mathrm{H}-3^{\prime}\right)$ & $6.86(\mathrm{~d}, \mathrm{~J}=2.4 \mathrm{~Hz}, 2 \mathrm{H}, \mathrm{H}-13)$ & $6.76(\mathrm{H}-5)$ \\
\hline \multicolumn{3}{|l|}{$6.98(1 \mathrm{H}, \mathrm{H}-13)$} \\
\hline \multicolumn{3}{|l|}{$6.95((\mathrm{~d}), 1 \mathrm{H}, \mathrm{H}-11)$} \\
\hline \multicolumn{3}{|l|}{$6.82((\mathrm{~d}), 1 \mathrm{H}, \mathrm{H}-3)$} \\
\hline \multicolumn{3}{|l|}{$6.72((d), 1 \mathrm{H}, \mathrm{H}-5)$} \\
\hline $4.48\left(\mathrm{t},{ }^{3} \mathrm{~J}=7.7 \mathrm{~Hz}, \mathrm{H}-1\right)$ & $4.74\left(\mathrm{t},{ }^{3} \mathrm{~J}=8.0 \mathrm{~Hz}, 1 \mathrm{H}, \mathrm{H}-1\right)$ & $4.58((\mathrm{t}), \mathrm{H}-1)$ \\
\hline $4.20\left(\mathrm{~d},{ }^{2} \mathrm{~J}=12.8 \mathrm{~Hz}, \mathrm{H}-9 \mathrm{a}\right)$ & $4.09\left(\mathrm{~d},{ }^{2} \mathrm{~J}=13.3 \mathrm{~Hz}, 1 \mathrm{H}, \mathrm{H}-17 \mathrm{a}\right)$ & 4.30 (br, H-9a) \\
\hline $3.87\left(\mathrm{~d},{ }^{2} \mathrm{~J} \sim 15.0 \mathrm{~Hz}, \mathrm{H}-17 \mathrm{a}\right)$ & $3.89\left(\mathrm{~d},{ }^{2} \mathrm{~J}=14.7 \mathrm{~Hz}, 2 \mathrm{H}, \mathrm{H}-9 \mathrm{a}\right)$ & 3.18 (br, H-9b) \\
\hline $3.86\left(\mathrm{~d},{ }^{2} \mathrm{~J}=13.5 \mathrm{~Hz}, \mathrm{H}-9 \mathrm{a}^{\prime}\right)$ & $3.76(\mathrm{~d}, 2 \mathrm{H}, \mathrm{H}-9 \mathrm{~b}+)$ & not discriminable \\
\hline \multicolumn{3}{|l|}{$3.82\left(\mathrm{~d},{ }^{2} \mathrm{~J}=14.1 \mathrm{~Hz}, \mathrm{H}-17 \mathrm{~b}\right)$} \\
\hline \multicolumn{3}{|l|}{$3.81\left(\mathrm{~d}^{2} \mathrm{~J}=13.7 \mathrm{~Hz}, \mathrm{H}-9 \mathrm{~b}\right)$} \\
\hline \multicolumn{3}{|l|}{$3.18\left(\mathrm{~d},{ }^{2} \mathrm{~J}=12.8 \mathrm{~Hz}, \mathrm{H}-9 \mathrm{~b}\right)$} \\
\hline $3.56(\mathrm{~s}, 3 \mathrm{H}, \mathrm{H}-8)$ & $3.12(\mathrm{~s}, 6 \mathrm{H}, \mathrm{H}-8)$ & $3.87(\mathrm{~s}, \mathrm{H}-8)$ \\
\hline 3.56 (s, 3H, H-8') & $2.89(\mathrm{~s}, 6 \mathrm{H}, \mathrm{H}-16)$ & $3.82(\mathrm{~s}, \mathrm{H}-16)$ \\
\hline \multicolumn{3}{|l|}{$3.41(\mathrm{~s}, 3 \mathrm{H}, \mathrm{H}-16)$} \\
\hline \multicolumn{3}{|l|}{$2.00\left(\mathrm{~s}, 3 \mathrm{H}, \mathrm{H}-16^{\prime}\right)$} \\
\hline $1.98(\mathrm{~m}, \mathrm{H}-18)$ & $1.86(\mathrm{~m}, \mathrm{H}-18)$ & $2.01(\mathrm{~m}, \mathrm{H}-18)$ \\
\hline $1.42(\mathrm{~m}, \mathrm{H}-19)$ & $1.42(\mathrm{~m}, \mathrm{H}-19)$ & not discriminable \\
\hline $1.36(\mathrm{~m}, \mathrm{H}-20)$ & not discriminable & \\
\hline \multicolumn{3}{|l|}{$1.26-1.24(\mathrm{~m}, \mathrm{H}-21 \mathrm{a}-\mathrm{d})$} \\
\hline \multicolumn{3}{|l|}{$1.35(\mathrm{~m}, \mathrm{H}-22)$} \\
\hline \multicolumn{3}{|l|}{$1.26(\mathrm{~m}, \mathrm{H}-23)$} \\
\hline \multicolumn{3}{|l|}{$0.86(\mathrm{t}, 3 \mathrm{H}, \mathrm{H}-24)$} \\
\hline $1.38\left(\mathrm{~s}, 9 \mathrm{H}, \mathrm{H}-12 \mathrm{~b}^{\prime}\right)$ & & \\
\hline
\end{tabular}


Table S4b. ${ }^{13} \mathrm{C}-\mathrm{NMR}$-spectrum of $\mathbf{5}$ in $\mathrm{CDCl}_{3}$ at $263 \mathrm{~K}$.

\begin{tabular}{|c|c|c|c|}
\hline \multicolumn{2}{|c|}{$\operatorname{paco}\left(\boldsymbol{\Lambda}, \mathbf{\Delta}^{\prime}\right)$} & 1,2-alternate $(0)$ & cone $(\mathrm{M})$ \\
\hline $154.81\left(\mathrm{C}_{\mathrm{i}}-15\right)$ & $154.97\left(\mathrm{C}_{\mathrm{i}}{ }^{-7}{ }^{\prime}\right)$ & $154.60\left(\mathrm{C}_{\mathrm{i}}-15\right)$ & $154.8\left(\mathrm{C}_{\mathrm{i}}-7\right)$ \\
\hline $154.54\left(\mathrm{C}_{\mathrm{i}}-7\right)$ & $154.54\left(\mathrm{C}_{\mathrm{i}}-15^{\prime}\right)$ & $153.63\left(\mathrm{C}_{\mathrm{i}}-7\right)$ & not discriminable \\
\hline $144.90\left(\mathrm{C}_{\mathrm{i}}-12\right)$ & $144.92\left(\mathrm{C}_{\mathrm{i}}-4^{\prime}\right)$ & $144.44\left(\mathrm{C}_{\mathrm{i}}-12\right)$ & not discriminable \\
\hline $144.50\left(\mathrm{C}_{\mathrm{i}}-4\right)$ & $143.24\left(\mathrm{C}_{\mathrm{i}}-12^{\prime}\right)$ & $143.99\left(\mathrm{C}_{\mathrm{i}}-4\right)$ & \\
\hline $136.90\left(C_{i}-2\right)$ & $138.04\left(\mathrm{C}_{\mathrm{i}}-2^{\prime}\right)$ & $138.15\left(\mathrm{C}_{\mathrm{i}}-2\right)$ & not discriminable \\
\hline $135.34\left(\mathrm{C}_{\mathrm{i}}-10\right)$ & $133.10\left(\mathrm{C}_{\mathrm{i}}-10^{\prime}\right)$ & $135.10\left(C_{i}-14\right)$ & \\
\hline $133.25\left(C_{i}-6\right)$ & $133.10\left(\mathrm{C}_{\mathrm{i}}-14^{\prime}\right)$ & $132.66\left(\mathrm{C}_{\mathrm{i}}-10\right)$ & \\
\hline $132.74\left(\mathrm{C}_{\mathrm{i}}-14\right)$ & $132.76\left(\mathrm{C}_{\mathrm{i}}-6^{\prime}\right)$ & $132.59\left(\mathrm{C}_{\mathrm{i}}-6\right)$ & \\
\hline $125.59(\mathrm{CH}, \mathrm{C}-13)$ & 126.33 (CH, C-13') & $126.10(\mathrm{CH}, \mathrm{C}-5)$ & 126.3 (br, C-11) \\
\hline $125.10(\mathrm{CH}, \mathrm{C}-11)$ & $126.25\left(\mathrm{CH}, \mathrm{C}-11^{\prime}\right)$ & $125.87(\mathrm{CH}, \mathrm{C}-11)$ & 124.5 (br, C-13) \\
\hline 124.47 (CH,C-5) & $125.34\left(\mathrm{CH}, \mathrm{C}-5^{\prime}\right)$ & $125.29(\mathrm{CH}, \mathrm{C}-13)$ & $124.0(\mathrm{br}, \mathrm{C}-5)$ \\
\hline $121.95(\mathrm{CH}, \mathrm{C}-3)$ & 121.90 (CH,C-3') & $122.10(\mathrm{CH}, \mathrm{C}-3)$ & $121.5(\mathrm{br}, \mathrm{C}-3)$ \\
\hline $61.27\left(\mathrm{CH}_{3}, \mathrm{C}-8\right)$ & $59.90\left(\mathrm{CH}_{3}, \mathrm{C}-8^{\prime}\right)$ & $60.63\left(\mathrm{CH}_{3}, \mathrm{C}-8\right)$ & $61.3(\mathrm{C}-8)$ \\
\hline $60.23\left(\mathrm{CH}_{3}, \mathrm{C}-16\right)$ & $58.35\left(\mathrm{CH}_{3}, \mathrm{C}-16^{\prime}\right)$ & $59.90\left(\mathrm{CH}_{3}, \mathrm{C}-16\right)$ & $61.3(\mathrm{C}-16)$ \\
\hline $35.50(\mathrm{CH}, \mathrm{C}-1)$ & & $34.56(\mathrm{CH}, \mathrm{C}-1)$ & $36.0(\mathrm{br}, \mathrm{C}-1)$ \\
\hline $36.79\left(\mathrm{CH}_{2}, \mathrm{C}-17\right)$ & $37.83\left(\mathrm{CH}_{2}, \mathrm{C}-9{ }^{\prime}\right)$ & $36.85\left(\mathrm{CH}_{2}, \mathrm{C}-9\right)$ & 31.0 (br, C-9) \\
\hline $30.98\left(\mathrm{CH}_{2}, \mathrm{C}-9\right)$ & & $30.06\left(\mathrm{CH}_{2}, \mathrm{C}-17\right)$ & not discriminable \\
\hline $33.88\left(\mathrm{CH}_{2}, \mathrm{C}-18\right)$ & & $32.69\left(\mathrm{CH}_{2}, \mathrm{C}-18\right)$ & not discriminable \\
\hline $28.46\left(\mathrm{CH}_{2}, \mathrm{C}-19\right)$ & & $28.10\left(\mathrm{CH}_{2}, \mathrm{C}-19\right)$ & \\
\hline $29.81\left(\mathrm{CH}_{3}, \mathrm{C}-20\right)$ & & not discriminable & \\
\hline $29.63,29.60,29.53$ & & & \\
\hline $29.34\left(\mathrm{CH}_{2}, \mathrm{C}-21 \mathrm{a}-\mathrm{d}\right)$ & & & \\
\hline $31.87\left(\mathrm{CH}_{2}, \mathrm{C}-22\right)$ & & & \\
\hline $22.67\left(\mathrm{CH}_{2}, \mathrm{C}-23\right)$ & & & \\
\hline $14.21\left(\mathrm{CH}_{3}, \mathrm{C}-24\right)$ & & & \\
\hline $33.87(\mathrm{C}, \mathrm{C}-4 \mathrm{a})$ & 33.87 (C,C-4a') & $34.02(\mathrm{C}, \mathrm{C}-4 \mathrm{a})$ & not discriminable \\
\hline 33.87 (C,C-12a) & 33.87 (C,C-12a') & $34.02(\mathrm{C}, \mathrm{C}-12 \mathrm{a})$ & \\
\hline $31.41\left(\mathrm{CH}_{3}, \mathrm{C}-12 \mathrm{~b}\right)$ & $31.51\left(\mathrm{CH}_{3}, \mathrm{C}-12 \mathrm{~b}^{\prime}\right)$ & $31.46\left(\mathrm{CH}_{3}, \mathrm{C}-4 \mathrm{~b}\right)$ & \\
\hline $31.25\left(\mathrm{CH}_{3}, \mathrm{C}-4 \mathrm{~b}\right)$ & $31.35\left(\mathrm{CH}_{3}, \mathrm{C}-4 \mathrm{~b}^{\prime}\right)$ & $31.36\left(\mathrm{CH}_{3}, \mathrm{C}-12 \mathrm{~b}\right)$ & \\
\hline
\end{tabular}


Table S5. Selected conformational parameters of the calixarene $2 \cdot \mathrm{CHCl}_{3}$.

\begin{tabular}{|c|c|}
\hline Compound & $2 \cdot \mathrm{CHCl}_{3}$ \\
\hline \multicolumn{2}{|c|}{ Interplanar angles $\left({ }^{\circ}\right)^{\mathrm{a}}$} \\
\hline $\mathrm{mpla} / \mathrm{A}$ & $83.68(1)$ \\
\hline $\mathrm{mpla} / \mathrm{B}$ & $43.73(1)$ \\
\hline $\mathrm{mpla} / \mathrm{C}$ & $87.43(1)$ \\
\hline mpla/D & $86.46(1)$ \\
\hline $\mathrm{A} / \mathrm{C}$ & $8.90(1)$ \\
\hline $\mathrm{B} / \mathrm{D}$ & $42.77(1)$ \\
\hline
\end{tabular}

${ }^{\mathrm{a}}$ Aromatic rings: ring $\mathrm{A}: \mathrm{C}(1) \ldots \mathrm{C}(6)$; ring $\mathrm{B}: \mathrm{C}(8) \ldots \mathrm{C}(13)$; ring $\mathrm{C}: \mathrm{C}(15) \ldots \mathrm{C}(20)$; ring $\mathrm{D}: \mathrm{C}(22) \ldots \mathrm{C}(27)$;
${ }^{\mathrm{b}}$ Best plane through atoms $\mathrm{C}(7), \mathrm{C}(14), \mathrm{C}(21)$ and $\mathrm{C}(28)$.

Table S6. Distances und angles of possible hydrogen-bond type interactions of 2.

\begin{tabular}{|c|c|c|c|c|}
\hline \multirow[t]{2}{*}{ Atoms involved } & \multirow[t]{2}{*}{ Symmetry } & \multicolumn{2}{|c|}{ Distances $(\AA)$} & \multirow{2}{*}{$\begin{array}{c}\text { Angles }\left(^{\circ}\right) \\
\text { D-H } \cdots A\end{array}$} \\
\hline & & $\mathrm{D} \cdots \mathrm{A}$ & $\mathrm{H} \cdots \mathrm{A}$ & \\
\hline C1G-H1G”'centroid(C) ${ }^{\mathrm{a}}$ & $\mathrm{x}, \mathrm{y}, \mathrm{z}$ & $3.8855(10)$ & $2.90(1)$ & 170 \\
\hline C33-H33A $\cdots{\text { centroid }(B)^{a}}^{a}$ & $-x, 1-y, 1 / 2+z$ & $3.4855(10)$ & $2.62(1)$ & 147 \\
\hline C1GA-H1GA $\cdots$ centroid $(C)^{\mathrm{a}}$ & $\mathrm{x}, \mathrm{y}, \mathrm{z}$ & $3.5692(10)$ & $2.63(1)$ & 157 \\
\hline
\end{tabular}

${ }^{a}$ Centroid means center of gravity of the respective aromatic ring: ring $\mathrm{A}: \mathrm{C}(1) \ldots \mathrm{C}(6)$; ring $\mathrm{B}: \mathrm{C}(8) \ldots \mathrm{C}(13)$; ring $\mathrm{C}$ : $\mathrm{C}(15) \ldots \mathrm{C}(20)$; ring D: $\mathrm{C}(22) \ldots \mathrm{C}(27)$. 